\title{
Flux Expulsion - Field Evolution in Neutron Stars
}

\author{
M. Jahan-Miri
}

Institute for Advanced Studies in Basic Sciences, Zanjan, 45195, IRAN

Received — 


\begin{abstract}
Models for the evolution of magnetic fields of neutron stars are constructed, assuming the field is embedded in the proton superconducting core of the star. The rate of expulsion of the magnetic flux out of the core, or equivalently the velocity of outward motion of flux-carrying proton-vortices is determined from a solution of the Magnus equation of motion for these vortices. A force due to the pinning interaction between the proton-vortices and the neutron-superfluid vortices is also taken into account in addition to the other more conventional forces acting on the proton-vortices. Alternative models for the field evolution are considered based on the different possibilities discussed for the effective values of the various forces. The coupled spin and magnetic evolution of single pulsars as well as those processed in low-mass binary systems are computed, for each of the models. The predicted lifetimes of active pulsars, field strengths of the very old neutron stars, and distribution of the magnetic fields versus orbital periods in low-mass binary pulsars are used to test the adopted field decay models. Contrary to the earlier claims, the buoyancy is argued to be the dominant driving cause of the flux expulsion, for the single as well as the binary neutron stars. However, the pinning is also found to play a crucial role which is necessary to account for the observed low field binary and millisecond pulsars.
\end{abstract}

Subject headings: magnetic fields - pulsars: general - stars: neutron - binaries: close 


\section{INTRODUCTION}

The observed high rate of incidence of binary pulsars among the population of low-magnetic field pulsars indicates a field decay mechanism in a neutron star which is linked with its evolution in a binary system (eg., Phinney \& Kulkarni 1994). This is also supported by even lower field strengths of the millisecond pulsars which are generally believed to be the end products of the binary evolution of pulsars in systems with low-mass main-sequence companions (eg., van den Heuvel 1994). A model for the evolution of the magnetic field in neutron stars has been proposed which indeed relates it to the spin evolution of the star, in an intimate way. The model predicts that the magnetic flux is transported out of the core, at the same rate as that of the spin-down of the star, into the crust where it undergoes Ohmic decay subsequently (Srinivasan et al. 1990). This field decay mechanism has been already tested in a study of the spin evolution of pulsars in binary systems. The distribution of the final field strengths against the orbital periods was found to be in good agreement with that of the observed wide-orbit low-mass binary pulsars (Jahan-Miri \& Bhattacharya 1994). Also, implications of the model for the evolution of single pulsars as well as those processed in massive binaries were shown to be consistent with, and provide new insights into, the existing observational data on the radio and X-ray pulsars (Jahan-Miri 1996a).

The above flux expulsion model (the so-called model of spin-down-induced flux expulsion, hereafter the SIF model) assumes that, as the star spins down, the outward moving (neutron) vortices pull the fluxoids (the proton-vortices) along with them because of their interpinning. However, given the finite strength of the pinning interaction energy and also the presence of resistance against the motion of both the fluxoids and the vortices the two might be, in general, expected to move with different radial velocities, while "cutting" or "creeping" through each other. A more refined treatment of the flux expulsion, therefore, requires the dynamics of fluxoid motion to be considered explicitly, as is attempted here. A further improvement, not addressed here, would be to solve simultaneously for the dynamics of the fluxoids and the vortices, self-consistently. Our treatment is, however, justified for the steady-state spin-down periods when the core superfluid spins down at a given rate which determines the radial velocity of the vortices.

In the following, we discuss the various forces which act on the fluxoids in the interior of a neutron star, including $i$ ) a force due to their pinning interaction with the moving vortices. The other forces which we take into account are ii) viscous drag force due to magnetic scattering of electrons, iii) buoyancy force, and $i v$ ) curvature force. The velocity of the outward motion of the fluxoids could be then determined, for a given steady-state spin-down rate of the star, from a solution of the Magnus equation requiring a balance of the forces acting radially on a fluxoid. The derived radial velocity of the fluxoids at the core-crust boundary would in turn determine the rate of the flux expulsion out of the core. Our approach here is analogous to that of Ding, Cheng \& Chau (1993, hereafter DC93) in their study of the field evolution of single normal pulsars (see also Muslimov \& Tsygan 1985; Harvey, Ruderman \& Shaham 1986; Jones 1987, 1991). The original motivation for the present work was to investigate the results of such a treatment of the flux expulsion scenario for the magnetic evolution of binary pulsars for which the spin-down history of the neutron star is quite different than that of the single pulsars addressed in DC93. Furthermore, we have also explored other possibilities different than those assumed by DC93 about the nature and magnitude of the various forces acting on the fluxoids. The resulting alternative models for calculating the rate of flux expulsion could, in principle, probe the physics of the interior of neutron stars in more details. Predictions of these models for the magnetic evolution of binary as well as single pulsars are compared and tested against the available observational data.

\section{Dynamics of Fluxoids}




\subsection{The Scene}

Superconductivity of the protons in the interior of a neutron star and the presence of a lattice of fluxoids carrying the magnetic flux in the core form the underlying premise for the field decay mechanism under study. As it is the case also with the assumed superfluidity of neutrons in a neutron star, the protons are speculated to form a (type II) superconductor based on the microscopic calculations of the associated energy gap in the BCS theory (Ruderman 1972). The corresponding transition temperature for the proton-superconductivity, though smaller than that of the neutron-superfluidity in the crust, is similar to that of the neutron-superfluidity in the core (se, eg., Sauls 1989) and much larger than the expected interior temperatures for the observed pulsars (Pines \& Alpar 1992). Doubts against superconductivity of the protons have been, however, raised because the earlier calculated core coupling time scale (Alpar \& Sauls 1988), for the case of superconductor protons, could not successfully accommodate the observed post-glitch recovery of the Vela pulsar (Pines \& Alpar 1992). This difficulty does not exists anymore since a necessary correction in the calculation of the coupling time scale results in more than an order of magnitude reduction in its value and makes it much smaller than the inferred observational upper limit (Jahan-Miri 1998). Moreover, the velocity relaxation time scale of the vortices for the case of normal protons in the core would be much larger than, or at the best (for the very cool neutron stars) similar to, the case of superconducting protons (see, eg., Sauls 1989). Hence normal protons may not offer any solution to the problem. On the other hand, the additional coupling mechanism due to an interpinning of the fluxoids and the vortices could result in a reduction in the velocity relaxation time of the vortices as well as in the coupling time for the superfluid bulk matter (estimates of the predicted time scales may be found in Jahan-Miri 1998). Thus superconductivity of the protons is rather favored by the above observational restriction on the core coupling time scale.

Given a superfluid-superconductor mixture in the interior of a neutron star with the associated (neutron) vortices and (proton) fluxoids, we further assume that both families of the vortex lines form regular uniform lattices parallel to the spin and magnetic axes, respectively. A stabilizing toroidal field component might be also present in the core fluid, should the crystalization of the crust does not take place early enough after the birth of a neutron star (Flowers \& Ruderman 1977). The toroidal component, if it is assumed to be $\left(>>10^{15} \mathrm{G}\right)$ much larger than the observable dipolar field would quench the superconductivity of the protons in the core, altogether. Otherwise, and if it is frozen, along with the dipolar field, within the superconducting core it would result in a complicated distorted geometry of the fluxoids. Such ditorted geometries of lines, which is neglected here for simplicity, would affect our following treatment of the flux expulsion in two ways. The effective total length of the fluxoids would be more than in the case of a regular parallel lattice assumed here. And, the entanglement of the fluxoids with the neutron vortices would be realized more effectively even in the cases when the rotation and magnetic axes of the star are nearlly parallel. The case of nearly parallel axes are indeed preferred in our models (see the next paragraph) thus the introduction of a distorted fluxoid geometry would have points for and against the following analysis. The rate of the Ohmic decay of the expelled flux in the crust is subject to uncertainties about the correct value of the conductivity of the crust matter as well as the geometry and transport behavior of the expelled flux due to other possible processes, namely the Hall drift and the turbulent cascade mechanism (see, eg., Jones 1988; Goldreich \& Reisenegger 1992; Bhattacharya \& Datta 1996). Different values for the effective decay time scale of the field in the crust are therefore tested in our model computations, to bypass these complications.

Throughout, we will be considering the motion of the fluxoids only in the region close to the core-crust boundary that also lies in the magnetic equatorial plane. The strength of the average field of the stellar core is decreased by the transport of these boundary fluxoids out of the core; the fluxoids in the interior regions are assumed to adjust their positions accordingly to maintain a uniform density. We will be referring to a cylindrical coordinate system aligned with the fluxoids in considering the radial and azimuthal directions, which coincide with their counterparts in a spherical system for the above region of interest. The radial 
velocities of the neutron vortices, in the same region, would however be co-linear with that of the fluxoids only for the vortex segments located near the spin equator of the star as well. The fractional size of such a region (of coincidence of the magnetic and spin equators) would be larger, hence our treatment of the radial velocities and forces for the fluxoids and the vortices would be more accurate, for the smaller inclination angles.

It has been suggested (Sauls 1989; Jones 1991) that, since the pinning interaction energy is independent of a displacement of a pinned neutron vortex parallel to itself, the vortices might be able to slide along the fluxoids without producing a large scale movement of the fluxoids. It is noted, however, that such a sliding might be realized only for the vortices in some parts of the spin equator, namely for those lying at large magnetic latitudes. Moreover, this possibility does not by itself violate our following assumption of a radial reactive force acting on the fluxoids even in the regions where sliding might occur. The Magnus force on a sliding vortex would have the same magnitude and direction as for the non-sliding vortices and its component in the direction perpendicular to the fluxoid has to be balanced in any case with a force due to the pinning which will exert a reaction force on the fluxoid. Furthermore, since the assumed sliding cannot be realized for all of the vortices, for any assumed geometry of the lines, its partial occurrence, if at all, seems to be further questionable on the account that it would result in an azimuthally non-uniform distribution of the vortex density.

\subsection{Neutron Superfluid Vortices}

In the steady-state, the vortices in the core of a neutron star are expected to be co-rotating with the charged component of the star, including the lattice of the proton fluxoids, at a given rate $\Omega$ (Sauls 1989). However, the superfluid bulk matter has to rotate faster at a rate $\Omega_{\mathrm{s}}$, maintaining a rotational lag

$\omega \equiv \Omega_{\mathrm{s}}-\Omega$ with its vortices, in order to follow the spinning down of the star at a rate $\dot{\Omega}$. The corresponding radial velocity $v_{\mathrm{n}}$ of the vortex outward motion, at the core boundary, would be

$$
\begin{aligned}
v_{\mathrm{n}} & =-\frac{R_{\mathrm{c}}}{2} \frac{\dot{\Omega}}{\Omega_{\mathrm{s}}} \approx-\frac{R_{\mathrm{c}}}{2} \frac{\dot{\Omega}}{\Omega} \\
& =1.59 \times 10^{-2} \frac{\dot{P}_{\mathrm{yr}}}{P_{\mathrm{s}}} \mathrm{cm} \mathrm{s}^{-1}
\end{aligned}
$$

where $R_{\mathrm{c}}=9 \times 10^{5} \mathrm{~cm}$ is the assumed radius of the core of a neutron star, and $P_{\mathrm{s}}$ and $\dot{P}_{\mathrm{yr}}$ are the spin period and its time derivative in units of $\mathrm{s}$ and $\mathrm{s} \mathrm{yr}^{-1}$, respectively. The superfluid spins down as the number of vortices is decreased due to their outward motion. The rotational lag $\omega>0$, in a spinning down superfluid, results in an outward radial Magnus force $F_{\mathrm{M}}=\rho_{\mathrm{s}} \kappa R_{\mathrm{c}} \omega$ on the vortices, per unit length at the core boundary, where $\rho_{\mathrm{s}}$ is the neutron-superfluid density, and $\kappa=2 \times 10^{-3} \mathrm{~cm}^{2} \mathrm{~s}^{-1}$ is the vorticity of a vortex line. The Magnus force is usually assumed to be balanced by the viscous force against the motion of the vortices which is primarily caused by the scattering of electrons off the magnetized cores of neutron vortices in the interior of a neutron star (Sauls 1989).

\section{The pinning force on the neutron vortices :}

On the other hand, if a pinning interaction is associated with an intersecting fluxoid-vortex pair the corresponding pinning force on the vortex could be, in principle, in either (inward or outward) radial directions. The force direction is decided by the direction of the relative motion of the interacting vortex and fluxoid, and is independent of the actual direction of motion of the vortices. That is, the pinning force could contribute to the viscous forces against the outward motion of the vortices if they move faster than the fluxoids. However, in the opposite case it would act as a "driving" force for the spinning down of the 
superfluid, being in the same direction as the outward motion of the vortices. Moreover, the viscous force due to the electron scattering is expected to be many orders of magnitudes smaller than the pinning force, even for the largest spin-down rates of interest and hence for the largest possible values of $v_{\mathrm{n}}$. Typically, the pinning force, per unit length of a vortex, is expected to be $>>10^{12} \mathrm{dyn}_{\mathrm{cm}}^{-1}$, while the viscous force of the electron scattering (Alpar \& Sauls 1988) is $<10^{5} \mathrm{dyn}^{-1}$ (and the buoyancy force on the magnetized neutron-vortices is even smaller than this). Therefore, for the vortices the pinning forces exerted by the fluxoids have to be balanced by the Magnus force on them.

An interesting consequence of such a balance of forces for the vortices is that the superfluid might be rotating slower than its vortices $(\omega<0)$ while it is spinning down. This is in contrast to the usual conditions during a spin-down phase of a superfluid, in general and, particularly when pinning is present and larger values of the lag are the case. As indicated, a spinning down superfluid must be rotating faster than its vortices $(\omega>0)$, so that the viscous force (as well as the pinning force if it is present) on the outward moving vortices is balanced by an outward driving Magnus force. However, since for the above assumed case the pinning force is itself directed outward it could as well play the role of the driving force, which in turn requires the balancing Magnus force to be directed inward. Thus, a negative rotational lag $\left(\omega=\Omega_{\mathrm{s}}-\Omega<0\right)$, hence an inward Magnus force, during a spin-down phase of the superfluid in the core of a neutron star is realized when the fluxoids move faster than the vortices.

\subsection{The Pinning Force on the Fluxoids}

In any case, a pinning force of the same magnitude as, and in the opposite direction to, that exerted by the fluxoids will be also exerted by the vortices on the fluxoids. Considering the above discussed balance of the forces on vortices, and equating the forces communicated between the two lattices of the vortices and the fluxoids, per unit volume, the pinning force $F_{\mathrm{n}}$ acting on a fluxoid, per unit length, is derived as

$$
\begin{aligned}
F_{\mathrm{n}} & =\frac{n_{\mathrm{v}}}{n_{\mathrm{f}}} F_{\mathrm{M}} \approx 2 \phi_{0} \rho_{\mathrm{s}} R_{\mathrm{c}} \frac{\Omega(t) \omega(t)}{B_{\mathrm{c}}(t)} \\
& =5.03 \frac{\omega_{-6}}{P_{\mathrm{s}} B_{8}} \text { dyncm }^{-1}
\end{aligned}
$$

where $n_{\mathrm{v}}=\frac{2 \Omega_{\mathrm{s}}}{\kappa}$ and $n_{\mathrm{f}}=\frac{B_{\mathrm{c}}}{\phi_{0}}$ are the number densities per unit cross section area of the vortices and the fluxoids, respectively, $\phi_{0}=2 \times 10^{-7} \mathrm{G} \mathrm{cm}^{2}$ is the magnetic flux carried by a fluxoid, $B_{\mathrm{c}}=10^{8} B_{8}$ is the strength of the core field in units of $\mathrm{G}$, and $\omega_{-6}$ is the superfluid lag $\omega$ in units of $10^{-6} \mathrm{rad} \mathrm{s}^{-1}$. Notice that the sign of $\omega$ determines the sign of $F_{\mathrm{n}}$ for which, as well as for the other forces discussed below, the outward direction will be reckoned as the positive sense.

The above derivation of $F_{\mathrm{n}}$ assumes that the total Magnus force acting continuously on all the vortices is communicated instantaneously to all the fluxoids. In contrast, it might be argued that in general only a small fraction of the vortices would be directly interacting with the fluxoids at any instant of time. The remaining much greater fraction of them (of the order of the ratio of an inter-fluxoid spacing to the size of a pinning interaction region) should reside in the inter-fluxoid spacings. Also, the assumed total force on the fluxoids has been divided equally among all in spite of the similar expectation that at any given time a majority of them would be located in the inter-vortex regions, far from any pinning site. Nevertheless, the motion of the fluxoids (as well as the vortices) is further constrained due to their mutual repulsive forces, which would require a uniform density of the lines to be maintained, in a steady state. Consequently, the fluxoids (whether being in an interaction region or in a free region) are forced to move always together (on scales of, at least, an inter-vortex spacing) which requires that the force acting on some of them to be shared equally among all, instantaneously, as is assumed in Eq. 2. The same argument fails however for the vortices since their displacements on scales of the order of an inter-fluxoid spacing (which is many 
orders of magnitudes smaller than the inter-vortex spacing) is not prohibited by the above requirement of having a uniform density. On the other hand, and for the same reason, any assumed vortex which is not interacting with a fluxoid at a given time is expected to move relative to the rest of the neighboring vortices and rapidly adjust its position (within a distance of an inter-fluxoid spacing) until it also is located within a pinning interaction zone. That is all the vortices would be continuously interacting with the fluxoids, which completes the justification of the above derivation of $F_{\mathrm{n}}$. Hence, Eq. 2 is valid during a vortex-fluxoid co-moving phase which was (implicitly) assumed in the above arguments.

However, should the (radial) velocities of vortices and fluxoids be different, the above restriction on the vortex positions might not be fulfilled steadily since, in this case, they have to continually move through the inter-fluxoids spacings as well. The effective instantaneous force per unit length of a fluxoid, $F_{\mathrm{n}}$, during such a state would be smaller than that given in Eq. 2, and could be estimated as the time averaged force acting on the fluxoids (or equivalently by using the fractional number of the vortices interacting with the fluxoids at any time). Therefore, during a phase of unequal velocities of the vortices and fluxoids one derives (in contrast to Eq. 2 for a co-moving phase)

$$
\begin{aligned}
F_{\mathrm{n}} & =\frac{d_{\mathrm{P}}}{d_{\mathrm{f}}}\left(\frac{n_{\mathrm{v}}}{n_{\mathrm{f}}} F_{\mathrm{M}}\right) \\
& =2.59 \times 10^{-4} \frac{\omega_{-6}}{P_{\mathrm{s}} B_{8}^{1 / 2}} \mathrm{dyn} \mathrm{cm}^{-1}
\end{aligned}
$$

where $d_{\mathrm{f}}=2.3 \times 10^{-7} B_{8}^{-\frac{1}{2}} \mathrm{~cm}$ is the inter-fluxoid spacing, and $d_{\mathrm{P}}$ is the effective size of a pinning interaction region around each fluxoid. A value of $d_{\mathrm{P}}=\lambda_{\mathrm{p}}^{*}=118 \mathrm{fm}$ has been used for the assumed magnetic pinning mechanism (see below), where $\lambda_{\mathrm{p}}^{*}$ is the effective London length of the proton superconductor, being also a length scale for the spread of the magnetic field of a neutron vortex line (Sauls 1989).

The "averaged" value of $F_{\mathrm{n}}$ as given in Eq. 3 assumes that the velocity of a vortex while it is crossing through an interaction region is the same as in the interaction-free space between the fluxoids; equal weights have been accordingly assigned to the corresponding time periods. Realistically, however, the vortices might be expected to move much faster in the free regions than in the pinning regions because of the large difference in the effective resisting forces acting on them in the two environments (as discussed above). As a consequence, they might tend to spend most of their time within the pinning zones which would require an almost zero weight to be assigned to the crossing times of the inter-fluxoid spacings. That is, their dynamics would resemble the case of the co-moving phase and, $F_{\mathrm{n}}$ as in Eq. 2 would be applicable even when there is a relative motion between the fluxoids and the vortices.

Thus, while for a co-moving phase $F_{\mathrm{n}}$ is uniquely determined by Eq. 2, when velocities are different one is faced with two different possibilities as given by Eq. 2 and Eq. 3. The two derivations represent, in fact, two extreme possibilities regarding the relative behavior of the different pinned segments of a vortex during its motion. If each pinned segment of a vortex could creep independently (over the length scales of about an inter-fluxoid spacing) then the above argument to justify Eq. 2 applies to the phase of nonequal velocities as well. In contrast, for a vortex line of infinite rigidity the whole line moves always as a single piece and $F_{\mathrm{n}}$ as in Eq. 3 would be appropriate. We will test both possibilities in the alternative models which we use, in order to distinguish the relevant approximation for the behavior of the vortices in the core of neutron stars.

\section{The "critical lag":}

The magnitude of the force which could be exerted at each intersection by a vortex on a fluxoid, and vice versa, is limited by a maximum value $f_{\mathrm{P}}$ corresponding to the given strength of the pinning energy $E_{\mathrm{P}}$ and the finite length scale of the interaction $d_{\mathrm{P}}$; namely $E_{\mathrm{P}}=f_{\mathrm{P}} d_{\mathrm{P}}$. The Magnus force on the vortices, which has to be balanced by the pinning force, (and hence $F_{\mathrm{n}}$ ) cannot therefore exceed a corresponding limit. This, in turn, implies a maximum critical lag $\omega_{\mathrm{cr}}$, which is determined by equating the Magnus force 
$\left(=\rho_{\mathrm{S}} \kappa R_{\mathrm{c}} \omega\right)$ with the maximum available pinning force $\left(=\frac{f_{\mathrm{P}}}{d_{\mathrm{f}}} \equiv \frac{E_{\mathrm{P}}}{d_{\mathrm{f}} d_{\mathrm{P}}}\right)$, per unit length of a vortex. The pinning energy, in the magnetic interaction mechanism, arises because of the difference in the free energies of a fluxoid-vortex pair when they overlap at an intersection or are separated. For a fluxoid with an average field $\vec{B}_{\mathrm{p}}$ and a vortex having an average field $\vec{B}_{\mathrm{n}}$, the magnetic energy density in the overlapping case would include a term $(2 / 8 \pi) \vec{B}_{\mathrm{p}} \cdot \vec{B}_{\mathrm{n}}$, in addition to the sum of their individual contributions, $\left(B_{\mathrm{p}}^{2}+B_{\mathrm{n}}^{2}\right) / 8 \pi$, when they are separated. The pinning energy, per intersection, is therefore estimated by multiplying the interaction overlap volume $\sim\left(2 \lambda_{\mathrm{p}}\right)\left(\pi \lambda_{\mathrm{p}}^{* 2}\right)$ with the above additional term of the energy density, which results in $E_{\mathrm{P}} \sim 10^{-5}$ ergs (see Jones 1991 for a more refined derivation). The critical lag $\omega_{\text {cr }}$ may be then calculated, as indicated above, to be

$$
\omega_{\mathrm{cr}}=1.59 \times 10^{-6} B_{8}^{1 / 2} \mathrm{rad} \mathrm{s}^{-1}
$$

where we have used the same parameter values as in DC93 in order for the further comparison of the results. The critical lag is the magnitude of the lag when velocities are different; $\omega=\omega_{\text {cr }}$ or $\omega=-\omega_{\text {cr }}$ when the vortices move faster or slower than the fluxoids, respectively. However, during a co-moving phase when the force communicated between a vortex and a fluxoid at each pinning point is less than its maximum value, $f_{\mathrm{P}}$, the lag might have any value within the range $-\omega_{\mathrm{cr}}<\omega<\omega_{\mathrm{cr}}$.

We note that a different estimate for the pinning energy due to the proton density perturbation gives a smaller value of $E_{\mathrm{P}} \sim 5 \times 10^{-7}$ ergs. The pinning energy in this case arises because of a difference in the condensation energy between the pinned and the free configurations, as a result of the change in the proton density induced by the large velocity of the neutrons close to the core of a neutron vortex (Sauls 1989). The interaction volume for this mechanism is $\sim \xi_{\mathrm{n}}^{2} \xi_{\mathrm{p}}$, and the change in the free energy density is estimated as $n_{\mathrm{n}} \frac{\Delta_{\mathrm{p}}^{2}}{E_{\mathrm{F}_{\mathrm{p}}}^{2}} \frac{\Delta_{\mathrm{n}}^{2}}{E_{\mathrm{F}_{\mathrm{n}}}^{2}}$, where $\Delta$ is the condensation energy gap, $E_{\mathrm{F}}$ is the Fermi energy, $\xi$ is the coherence length, $n$ is the number density, and the subscripts "p" and "n" refer to the protons and neutrons, respectively. The larger values of $E_{\mathrm{P}}$ in the case of the density perturbation is, however, associated with an interaction length, $d_{\mathrm{P}}=\xi_{\mathrm{p}}$, which is smaller than for the magnetic interaction, $\lambda_{\mathrm{p}}^{*}$, by about the same ratio as the inverse of the pinning energies. Thus, similar values of $f_{\mathrm{P}}$, and hence $\omega_{\mathrm{cr}}$, are expected for the both pinning mechanisms, as has been indicated earlier (Bhattacharya and Srinivasan 1991). Nevertheless, since the magnetic interaction depends on the angle between fluxoids and vortices the pinning caused by the density perturbation might indeed have the dominant effect in a neutron star with a nearly parallel geometry of the vortices and fluxoids.

\subsection{Other Forces on the Fluxoids}

In addition to the pinning force, the fluxoids in the interior of a neutron star are also subject to the following radial forces.

\section{The drag force:}

An isolated fluxoid moving through the normal degenerate electron gas in the core of a neutron star is subject to the viscous drag force of the electrons scattering off its magnetic field. The viscous drag force, per unit length of a fluxoid, is estimated (Jones 1987) to be

$$
\begin{aligned}
\vec{F}_{v} & =-\frac{3 \pi}{64} \frac{n_{\mathrm{e}} e^{2} \phi_{0}^{2}}{E_{\mathrm{F}_{\mathrm{e}}} \lambda_{\mathrm{p}}} \frac{\overrightarrow{v_{\mathrm{p}}}}{c} \\
& =-7.30 \times 10^{7} \overrightarrow{\mathrm{p}} \text { dyn } \mathrm{cm}^{-1}
\end{aligned}
$$

where $v_{\mathrm{p}}$ is the velocity of the outward radial motion of the fluxoids in units of $\mathrm{cm} \mathrm{s}^{-1}, n_{\mathrm{e}}=3 . \times 10^{36} \mathrm{~cm}^{-3}$ 
is the number density of the electrons, and $E_{\mathrm{F}_{\mathrm{e}}}=88 \mathrm{MeV}$ is the electron Fermi energy, corresponding to a total density $\rho=2 \times 10^{14} \mathrm{~g} \mathrm{~cm}^{-3}$, and a neutron number density $n_{\mathrm{n}}=1.7 \times 10^{38} \mathrm{~cm}^{-3}$ in the core.

The expression for $F_{v}$ in Eq. 5 is derived based on the assumption of independent motions for single fluxoids. However, for the typical conditions in the interior of a neutron star the lattice of fluxoids might be, as a whole (or at least as bundles consisting of not less than ten million fluxoids), "frozen-in" the electron gas (Harvey et al. 1986). This is because the mean free path of the electrons turns out to be many orders of magnitudes larger than the mean distance between their successive scattering events, and also the deflection angle at each event is very small. A treatment of the coherent electron scattering by the fluxoid lattice has been shown to indeed require an almost zero relative velocity between the electrons and the lattice (Jones 1987). Therefore, the flux expulsion out of the core might be prohibited except for the presence of electron-current loops across the core-crust boundary. Uncertainties about the true distribution of the magnetic flux and the correct value of the conductivity in the crust, and also the possibility of a mechanical failure of the solid crust due to a build-up of the magnetic stresses, however, obscure any definite conclusion to be drawn.

Moreover, there are other reasons to suspect the suggested frozen-in approximation for the fluxoids as a whole (see also DC93; Ruderman 1995), in the core of a neutron star. For example, the finite volume of the fluxoid lattice and also the influence of the superconductor boundary effects on the motion of the fluxoids which have not been included in the previous studies of the coherent scattering could as well have significant new consequences. In addition, the motion of the incompressible electron fluid in the interior of a neutron star has been argued to be divergence free (Goldreich \& Reisenegger (1992). The above permitted motion of the fluxoids, along with the electrons in the frozen-in approximation, must be, therefore, of the same (divergence free) nature. This is however impossible for the uniform lattice of fluxoids during its outward motion since its lattice constant keeps changing. Hence, a compromise between the flux freezing and the divergence free motion of the electrons has to be worked out if any flux expulsion is to be accounted for.

Given the above uncertainties as well as the lack of any other definite prescription for calculating the drag force due to the electron scattering, we use a value of $F_{v}$ as given by Eq. 5 in our models. This choice is also supported by noticing that a tentative expulsion time scale derived for the case of coherent scattering, as implied by the Hall drift of the flux in the base of the crust (Jones 1988, 1991), turns out to be similar to that based on the single fluxoid approximation. On the other hand, a maximum velocity for the outward motion of the fluxoids has been suggested for the case of coherent electron scattering (Jones 1991). Also, in some of our models (models B1 and B2, described below) a similar value for the maximum possible velocity of the fluxoids is adopted, eventhough for a different reason. The same models might be therefore viewed also as models representing the frozen-in approximation due to the coherent electron scattering.

\section{The buoyancy force:}

The buoyancy force on fluxoids in a neutron star arises for reasons analogous to the case of macroscopic flux tubes in ordinary stars. Because flux tubes are in pressure equilibrium with their surrounding the excess magnetic pressure causes a deficit in the thermal pressure, and hence in the density, of the plasma inside a flux tube which make the tube to become buoyant. The radially outward buoyancy force $F_{\mathrm{b}}$ on a fluxoid, per unit length, can be expressed as (Muslimov \& Tsygan 1985; Jones 1987)

$$
\begin{aligned}
F_{\mathrm{b}} & =\left(\frac{\phi_{0}}{4 \pi \lambda_{\mathrm{p}}}\right)^{2} \frac{\ln \left(\lambda_{\mathrm{p}} / \xi_{\mathrm{p}}\right)}{R_{\mathrm{c}}} \\
& =0.51 \mathrm{dyn} \mathrm{cm}^{-1}
\end{aligned}
$$

where values of $\lambda_{\mathrm{p}}=131.5 \mathrm{fm}$ and $\frac{\lambda_{\mathrm{p}}}{\xi_{\mathrm{p}}}=\sqrt{2}$ have been used.

Harrison (1991) has raised objection against the relevance of the buoyancy force for the motion of single fluxoids in the core of neutron stars, assuming the whole lattice of the fluxoids to be frozen in the 
electron-proton plasma within the star. He argues that the buoyancy force would rather contribute to the gradient of the macroscopic magnetic stresses supporting the hydrostatic equilibrium of the plasma within the star, instead of acting on the fluxoids individually. His argument is not, however, applicable if a relative motion between the fluxoids and the plasma is allowed to take place, as he also makes it clear (see, in particular, the last paragraph before $\S 3.2$, p. 422, in Harrison 1991). Thus, for our model calculations, having assumed a drag force corresponding to the single fluxoid motion, we also take into account the buoyancy force on the fluxoids, for self-consistency. In this context, Ding et al. (DC93) have argued that the above Harrison's objection against the buoyancy force might be irrelevant because the fluxoids motion could be fast enough such that the conditions of hydrostatic equilibium of the star is not satisfied during their motion. Such an argument is apparently missing the point, given that the independent motions of single fluxoids has been also assumed in DC93. The decision whether the buoyancy force acts on the fluxoid lattice as a whole, hence contributing to the hydrostatic pressure support, or else on each flux line individually is independent of, and would be equally effective in, the presence or absence of the equilibrium. Even if hydrostatic equilibrium within a neutron star is assumed, as is speculated in DC93, to be approached on timescales $>1 \mathrm{Myr}$, corresponding to the largest fluxoid velocities $<10^{-7} \mathrm{~cm} \mathrm{~s}^{-1}$, the buoyancy force could be still acting on the whole lattice, should a relative motion between the fluxoids and the plasma being prohibited due to the requirements of the electron scattering orbits off fluxoids.

\section{The curvature force :}

The tension of a vortex line (such as a fluxoid) implies that a curved geometry of the line would result in a restoring force, the curvature force, which tries to bring the line back to its minimum energy straight configuration. The concavely directed curvature force $F_{\mathrm{c}}$, per unit length, on a vortex having a tension $T$ and a curvature radius $S$ is given as $F_{\mathrm{c}}=T / S$ (Harvey et al. 1986). Thus, for a fluxoid in a neutron star, having a tension $T_{\mathrm{p}}=\left(\frac{\phi_{0}}{4 \pi \lambda_{\mathrm{p}}}\right)^{2} \ln \left(\lambda_{\mathrm{p}} / \xi_{\mathrm{p}}\right)$, the magnitude of the curvature force would be

$$
\begin{aligned}
\left|F_{\mathrm{c}}\right| & =\frac{R_{\mathrm{c}}}{S}\left(\frac{\phi_{0}}{4 \pi \lambda_{\mathrm{p}}}\right)^{2} \frac{\ln \left(\lambda_{\mathrm{p}} / \xi_{\mathrm{p}}\right)}{R_{\mathrm{c}}} \\
& \equiv \frac{R_{\mathrm{c}}}{S} F_{\mathrm{b}}
\end{aligned}
$$

where we have used Eq. 6 to express the absolute value of $F_{\mathrm{c}}$ in terms of the (positive) buoyancy force. Moreover, the end points of a fluxoid, where its magnetic flux spouts out and joins the almost uniform field of the crust, are expected to be frozen in at the bottom of the crust due to the large conductivity of the matter. An outward moving fluxoid might be therefore expected to bend outward and becomes subject to an inward curvature force, per unit length,

$$
\begin{aligned}
F_{\mathrm{c}} & =-\frac{R_{\mathrm{c}}}{S_{\mathrm{c}}} F_{\mathrm{b}} \\
& =-0.35 \mathrm{dyn} \mathrm{cm}^{-1}
\end{aligned}
$$

using Eq. 6, and a value of $\frac{R_{\mathrm{c}}}{S} \sim \ln 2$ for the assumed spatially uniform distribution of the fluxoids (DC93).

The currents at the bottom of the crust are however subject to diffusion processes, the rate of which would set a maximum limiting speed $v_{\max }$ for the motion of the end points of the fluxoids. We, therefore, assume that whenever $v_{\mathrm{p}}<v_{\max }$ the fluxoids remain straight and no curvature force will be acting on them $\left(F_{\mathrm{c}}=0\right)$, since their end points are also able to move with the same speed. In the opposite case, when $v_{\mathrm{p}} \geq v_{\max }$, the fluxoids would be bent outward and the force $F_{\mathrm{c}}$ as in Eq. 8 will be used. We note that a self-consistent solution of the equation of motion (Eq. 12 below) requires further that during a transition between the above two regimes $F_{\mathrm{c}}$ should change gradually (see Fig. 3 below). We have allowed for such a smooth variation of $F_{\mathrm{c}}$ at and around $v_{\mathrm{p}}=v_{\max }$, in the models which, otherwise, use the above prescription 
for calculating $F_{\mathrm{c}}$. The above maximum drift velocity $v_{\max }$ of the magnetic flux in the crust is estimated, based on the Ohmic diffusion alone, as

$$
\begin{aligned}
v_{\max } & \sim \frac{R}{\tau} \\
& =3.18 \times 10^{-9}\left(\frac{\tau}{10^{7} \mathrm{yr}}\right)^{-1} \mathrm{~cm} \mathrm{~s}^{-1}
\end{aligned}
$$

where $R=10^{6} \mathrm{~cm}$ is the radius of a neutron star, and $\tau$ is the assumed time scale, in units of yr, for the decay of the magnetic field in the crust. A larger value for $v_{\max }$ may be expected if the Hall drift of the magnetic flux at the bottom of the crust is also taken into account. Also, the mechanical failure, and the plate tectonic motion, of the crust (Ruderman 1991) may require a larger value of $v_{\text {max }}$, provided the plates motion is driven by some other cause rather than the pull of the fluxoids on the crust.

In contrast to the above velocity based criterion for deciding the sense of the bending of the fluxoids, DC93 assume that the fluxoids would be bent outward, and become subject to the same inward force $F_{\mathrm{c}}$ as

in Eq. 8, throughout the period of time $t<\tau$. They also assume that for times $t>\tau$ an outward force of a comparable magnitude would be effective. The latter (outward) force will be however ignored in our new models, since it implies an spontaneous motion of the end points of a fluxoid at a speed faster than the fluxoid itself which we don't find it to be justified. The tendency of a flux tube for decreasing its length under the effect of its tension (Harvey et al. 1986), which could result in an inward bending, might be of no consequence in the present case. Because, given the negligible radius of a fluxoid as compared to the radius of curvature of the core-crust boundary surface, a fluxoid might not be subject to any instantaneous force pulling it out to a position with a shorter length; it may remain in its "meta-stable" state being "unaware" of the possible gain in its free energy upon an outward displacement.

On the other hand, the value of $F_{\mathrm{c}}$ as given by Eq. 8, for conditions of $v_{\mathrm{p}}>v_{\text {max }}$, might be an underestimate. It has been argued that the repulsive force between the fluxoids should ensure that the lattice response to a deformation is determined, to a first approximation, by their collective rigidity (Jones 1991). The force $F_{\mathrm{c}}$ associated with even a piece of the lattice of a size of an inter-vortex spacing (including some $10^{7}$ flux lines) would be, in this approximation, so large that any bending of the lattice is effectively prohibited. The velocity of the fluxoids would be therefore constrained at all times by the condition $v_{\mathrm{p}} \leq v_{\max }$. In order to implement this latter assumption we, therefore, construct models which use the following prescription for calculating $F_{\mathrm{c}}$. If a value of $F_{\mathrm{c}}=0$ results in deriving $v_{\mathrm{p}}>v_{\max }$ (see below) then $v_{\mathrm{p}}=v_{\max }$ is assumed (otherwise, we set $F_{\mathrm{c}}=0$, and the derived value for $v_{\mathrm{p}}$ is used, as before), and $F_{\mathrm{c}}$ is calculated from

$$
F_{\mathrm{c}}=-\left(F_{\mathrm{n}}+F_{\mathrm{b}}+F_{v}\right)
$$

where the right hand side is evaluated using $v_{\mathrm{p}}=v_{\max }$, together with $\omega=\omega_{\mathrm{cr}}$ or $=-\omega_{\mathrm{cr}}$, whichever may be the case. As indicated earlier, these latter models might be alternatively viewed as representatives of the case of coherent electron scattering.

\section{The Models}

The steady-state radial motion of a fluxoid, in the region of interest, is thus determined from the balance equation for all the radial forces acting on it, per unit length, that is:

$$
F_{\mathrm{n}}+F_{v}+F_{\mathrm{b}}+F_{\mathrm{c}}=0
$$


Substituting in the above equation for the different forces from Eqs 2 or 3, 5, 6, and 8 or 10, respectively, it may be rewritten in the form

$$
\alpha \frac{\omega_{-6}}{P_{\mathrm{s}} B_{8}}-\beta v_{\mathrm{p}_{7}}+\delta=0
$$

where parameters $\alpha, \beta$, and $\delta\left(\equiv F_{\mathrm{b}}+F_{\mathrm{c}}\right)$ are given below for the different models, and $v_{\mathrm{p}_{7}}$ is the fluxoid velocity $v_{\mathrm{p}}$ in units of $10^{-7} \mathrm{~cm} \mathrm{~s}^{-1}$. Recall that $\omega_{-6}$, which is the value of $\omega$ in units of $10^{-6} \mathrm{rad} \mathrm{s}^{-1}$, might have either positive or negative values, as is also the case with $\delta$ in some of the models.

This single equation includes two unknown variables $\omega$ and $v_{\mathrm{p}}$, and represents the azimuthal component of the Magnus equation of motion (Sonin 1987) for the proton vortices. No radial Magnus force acts on the fluxoids, hence the right hand side is set to zero, because of the assumed co-rotation of the fluxoids with the proton superconductor. There exist however additional restrictions on the motion of the fluxoids which can be used to fix the value of one of the variables and solve Eq. 12 for the other. Namely, for a co-moving state $v_{\mathrm{p}}=v_{\mathrm{n}}$ is given and $\omega$ could be determined. In contrast, when $v_{\mathrm{p}}\left(\neq v_{\mathrm{n}}\right)$ is unknown $\omega$ is given as $\omega=\omega_{\mathrm{cr}}$ or $\omega=-\omega_{\text {cr }}$ for $v_{\mathrm{p}}<v_{\mathrm{n}}$ or $v_{\mathrm{p}}>v_{\mathrm{n}}$, respectively. Furthermore, inspection of Eq. 12 indicates that it admits one and only one of the three different solutions, for the given values of $v_{\mathrm{n}}, B_{\mathrm{c}}$, and $P_{\mathrm{s}}$ at any time, namely

$$
\begin{array}{llc}
\omega=\omega\left(v_{\mathrm{p}}=v_{\mathrm{n}}\right) & \text { iff } & -\omega_{\mathrm{cr}}<\omega<\omega_{\mathrm{cr}} \\
v_{\mathrm{p}}=v_{\mathrm{p}}\left(\omega=\omega_{\mathrm{cr}}\right) & \text { iff } & v_{\mathrm{p}}<v_{\mathrm{n}} \\
v_{\mathrm{p}}=v_{\mathrm{p}}\left(\omega=-\omega_{\mathrm{cr}}\right) & \text { iff } & v_{\mathrm{p}}>v_{\mathrm{n}}
\end{array}
$$

The rate of the flux expulsion out of the core, $\dot{B}_{\mathrm{c}}=-\frac{2}{R_{\mathrm{c}}} B_{\mathrm{c}} v_{\mathrm{p}}$, and the evolution of the stellar surface field $B_{\mathrm{s}}$ (with a decay rate $\dot{B}_{\mathrm{s}}=-\frac{B_{\mathrm{s}}-B_{\mathrm{c}}}{\tau}$ ) are hence uniquely determined from the above force balance equation, given the spin evolution of the star which determines (Eq. 1) the vortex velocity $v_{\mathrm{n}}$ at any time.

We construct four separate models, labeled A1, A2, B1, and B2, based on the two alternative estimates discussed earlier for the pinning force $F_{\mathrm{n}}$ and also for the curvature force $F_{\mathrm{c}}$, by permutation. The models are summarized below by indicating to which of the four distincting physical assumptions they relate, and also giving their associated values of the parameters in the force equation (Eq. 12).

- Model A1: $\left\{\begin{array}{l}\text { - vortex segments creep independently } \\ \text { - fluxoids may bend when } v_{\mathrm{p}}>v_{\max }\end{array}\right.$ $\alpha=5.03$,

$\delta= \begin{cases}0.51 & \text { if } v_{\mathrm{p}}<v_{\max } \\ 0.16 & \text { if } v_{\mathrm{p}} \geq v_{\max }\end{cases}$

- Model A2: $\left\{\begin{array}{l}\text { - vortices remain straight while moving } \\ \text { - fluxoids may bend when } v_{\mathrm{p}}>v_{\max }\end{array}\right.$ $\alpha=2.587 \times 10^{-4} B_{8}^{\frac{1}{2}}$,

$\delta=$ same as for $\mathrm{A} 1$

- Model B1: $\left\{\begin{array}{l}\text { - vortex segments creep independently } \\ \text { - fluxoids remain straight, constrained by } v_{\mathrm{p}} \leq v_{\max }\end{array}\right.$ 


$$
\begin{aligned}
& \alpha=\text { same as for A1, } \\
& \delta=\left\{\begin{array}{lll}
0.51 & \text { if } v_{\mathrm{p}}<v_{\max } \\
-\left(F_{\mathrm{n}}+F_{v}\right) & \text { if } v_{\mathrm{p}}=v_{\max }
\end{array}\right.
\end{aligned}
$$

- Model B2: $\left\{\begin{array}{l}\text { - vortices remain straight while moving } \\ \text { - fluxoids remain straight, constrained by } v_{\mathrm{p}} \leq v_{\max }\end{array}\right.$

$\alpha=$ same as for A2,

$\delta=$ same as for B1

while $\beta=7.30$ is the same for all the models

These four models together with the model adopted by DC93 (the DCC model) will be referred to collectively as the FBE models (those which employ a Force Balance Equation), in contrast to the SIF model which has a different approach for calculating the rate of flux expulsion. Table 1 compares all the six models by indicating how the forces and the fluxoid velocity are determined in each of them. Spin and magnetic evolution of the single as well as binary pulsars are calculated according to the requirements of each of the six models, separately, and the results are discussed in the following sections.

\begin{tabular}{|c|c|c|c|c|c|c|}
\hline \multicolumn{2}{|c|}{ model } & $F_{\mathrm{n}}$ & $F_{\mathrm{b}}$ and $F_{\mathrm{v}}$ & \multicolumn{2}{|c|}{$F_{\mathrm{c}}$} & $v_{\mathrm{p}}$ and $\omega$ \\
\hline \multirow{5}{*}{ FBE } & $\mathrm{DCC}$ & \multirow{3}{*}{ as in Eq. 2} & \multirow{3}{*}{ as in } & $\begin{array}{c}\text { as in Eq. } 8 \\
=\frac{\pi}{4} F_{\mathrm{b}}\end{array}$ & $\begin{array}{l}\text { if } t<\tau \\
\text { if } t \geq \tau\end{array}$ & \multirow{2}{*}{$\begin{array}{l}\text { from Eq. } 12, \\
\text { subject to : }\end{array}$} \\
\hline & A1 & & & $\begin{array}{c}\quad=0 \\
\text { as in Eq. } 8\end{array}$ & $\begin{array}{l}\text { if } v_{\mathrm{p}}<v_{\max } \\
\text { if } v_{\mathrm{p}} \geq v_{\max }\end{array}$ & \\
\hline & B1 & & & $\begin{array}{l}\quad=0 \\
\text { as in Eq. } 10\end{array}$ & $\begin{array}{l}\text { if } v_{\mathrm{p}}<v_{\max } \\
\text { if } v_{\mathrm{p}}=v_{\max }\end{array}$ & $v_{\mathrm{p}}=v_{\mathrm{n}} \quad$ iff $|\omega|<\omega_{\mathrm{cr}}$ \\
\hline & B2 & \multirow{2}{*}{$\begin{array}{l}\text { as in Eq. } 2 \text { if } v_{\mathrm{p}}=v_{\mathrm{n}} \\
\text { as in Eq. } 3 \text { if } v_{\mathrm{p}} \neq v_{\mathrm{n}}\end{array}$} & \multirow[t]{2}{*}{5 and 6} & \multicolumn{2}{|c|}{$\left(v_{\mathrm{p}}>v_{\max }\right.$ not permitted $)$} & \multirow{2}{*}{$\begin{array}{c}\omega=\omega_{\mathrm{cr}} \quad \text { iff } v_{\mathrm{p}}<v_{\mathrm{n}} \\
\omega=-\omega_{\mathrm{cr}} \quad \text { iff } v_{\mathrm{p}}>v_{\mathrm{n}}\end{array}$} \\
\hline & $\mathrm{A} 2$ & & & $\begin{array}{c}\quad=0 \\
\text { as in Eq. } 8\end{array}$ & $\begin{array}{l}\text { if } v_{\mathrm{p}}<v_{\max } \\
\text { if } v_{\mathrm{p}} \geq v_{\max }\end{array}$ & \\
\hline \multicolumn{2}{|c|}{ SIF } & \multicolumn{5}{|c|}{$v_{\mathrm{p}}=v_{\mathrm{n}}($ assumed $)$} \\
\hline
\end{tabular}

\section{Table 1- Different models of flux expulsion}

\section{Single Pulsars}

The spin evolution of a solitary pulsar driven by its electromagnetic torque has a rate $\dot{P}_{\mathrm{s}}=3.15 \times 10^{-32} \frac{B_{\mathrm{s}}^{2}}{P_{\mathrm{s}}} \mathrm{s} \mathrm{yr}^{-1}$, where $B_{\mathrm{s}}$ is the surface field in units of $\mathrm{G}$, and $P_{\mathrm{s}}$ is in units of seconds. From the instantaneous value of the spin-down rate one finds the velocity $v_{\mathrm{n}}$ of the outward motion 
of the neutron vortices (Eq. 1). Also, the critical lag $\omega_{\text {cr }}$ may be determined, from Eq. 4, for any given value of the core field strength $B_{\mathrm{c}}$. The solution of Eq. 12, for each model, given the values of $v_{\mathrm{n}}$ and $\omega_{\mathrm{cr}}$ at a time $t$, then determines the corresponding values of the fluxoids outward radial velocity $v_{\mathrm{p}}$ and the lag $\omega$ between the rotation rates of the vortices and the neutron superfluid in the core of the evolving solitary pulsar. The coupled evolution of the spin period and the magnetic field, in the core and at the surface, are thus followed over a period of $10^{10} \mathrm{yr}$ in order to cover both the young and the very old neutron stars in the Galaxy.

The computed time evolution of $v_{\mathrm{p}}$ and $\omega$ are shown in Fig. 1, together with $v_{\mathrm{n}}$ and $\omega_{\mathrm{cr}}$, as is predicted in the A1 model. Characteristically similar results as in Fig. 1 are obtained for the other FBE models as well. The evolution of the lag $\omega$ although is not directly of interest for our present analysis of the field evolution nevertheless bears significant consequences for an understanding of the rotational dynamics of neutron stars, to be discussed elsewhere.

The fluxoids motion in Fig. 1 is seen to follow three evolutionary phases in which they move slower, together, and faster than the vortices, successively. These will be referred to as forward creep, co-moving, and reverse creep phases, respectively (we use the terminology of DC93). Transitions between these successive evolutionary phases occur because of the reduction in $v_{\mathrm{n}}\left(\propto \dot{\Omega}_{\mathrm{s}}\right)$ as well as the increase in $P_{\mathrm{s}}$, and a final co-moving phase might also occur for some choices of the initial conditions. Note that $\omega$ changes sign from positive to negative and remains so in the later parts of the co-moving phase, as well as during the reverse creep phase. Also note that $|\omega|=\omega_{\text {cr }}$ during both the forward and the reverse creeping phases.

\subsection{Field Evolution}

The predicted evolution of the core and surface fields for a single neutron star according to the A1 model is given in Fig. 2; the other FBE models produce similar results. The two panels in Fig. 2 are for two different assumed values of the decay time scale $\tau$ in the crust, where Fig. 2a corresponds to the results in Fig. 1. A substantial decrease in the core field occurs at a time $t \gtrsim 10^{7} \mathrm{yr}$, which is expected for the typical average values of $v_{\mathrm{p}} \lesssim 10^{-8} \mathrm{~cm} \mathrm{~s}^{-1}$ during the earlier times, because $\frac{\dot{B}_{\mathrm{c}}}{B_{\mathrm{c}}}=\frac{v_{\mathrm{p}}}{R_{\mathrm{c}}}$ implies that a time period $\Delta t \sim \frac{R_{\mathrm{c}}}{v_{\mathrm{p}}}$ is needed for a major reduction in the core field to occur. However, because of the very small magnitude of $v_{\mathrm{p}}$ (although $\gtrsim v_{\mathrm{n}}$ ) and also the reduced value of $B_{\mathrm{c}}$ at later times $B_{\mathrm{c}}$ does not change, substantially, afterwards. The surface field $B_{\mathrm{s}}$ responds to the change in $B_{\mathrm{c}}$ on the assumed decay time scale $\tau$ of the crust. The nontrivial role of the stellar crust in these field evolution models may be seen by comparing Fig. 2a with Fig. 2b, where values of $\tau=10^{7}$, and $10^{8}$ yr have been used, respectively. A larger value of $\tau$ tends to maintain the initial $B_{\mathrm{s}}$, hence a larger $\dot{P}_{\mathrm{s}}$ as well as a larger $v_{\mathrm{n}}$, over a more extended period of time. Consequently, smaller final values of $B_{\mathrm{c}}$ and $B_{\mathrm{s}}$ are predicted for the larger assumed values of $\tau$, as is seen in Fig. 2.

Fig. 2a might seem to suggest that $B_{\mathrm{c}}$ stops decaying once $B_{\mathrm{s}}$ starts to decline, as is also concluded by DC93. However, this is but an artifact of the assumed value of $\tau$ which happens to be close to the saturation time of the core field decay. The spurious nature of such a correlation, that a decrease in the strength of the crustal field results in a halt in the decay of the core field, may be confirmed by using tentatively smaller values of $\tau \lesssim 10^{6} \mathrm{yr}$. As we have verified, in such cases the core and the surface fields are seen to decay simultaneously (see Fig. 3.3 in Jahan-Miri 1996b), in contrast to the above unreal correlation. The decay of the core field, according to the present models, does not have a direct and one-to-one dependence on that of the crust; instead they are coupled through the influence of many factors (see the preceeding paragraph). This statement may be also appreciated by contrasting the predicted field evolution under distinct intial conditions for the relative fields of the core and that of the crust, $B_{\text {crust. }}$. For this purpose, the results in Fig. 2 with an assumed intial condition $B_{\text {crust }} \sim 0.1 B_{\text {c }}$ may be compared with that in DC93 
where $B_{\text {crust }}=1.5 B_{\mathrm{c}}$ and also $B_{\text {crust }} \sim 5 \times 10^{3} B_{\text {c }}$ have been used (their Figs 2 and 3 ). The initial crustal field in our case compares with a very late stage of evolution in DC93 when the crustal field has been almost completely decayed. Hence, the initial core field in Fig. 2 should have not decayed, from the very beginning, if the above effect were real. The above differecnce in the adopted initial fields might deserve a further note. For an initially uniform distribution of the magnetic flux within the star, which is assumed in DC93 as well as here, the initial condition $B_{\text {crust }}=1.5 B_{\mathrm{c}}$ implies a relative radial size for the crust larger than the currently used values (eg., Sauls 1989; Pines \& Alpar 1992). It is particularly questionable that the same condition has been used in DC93 for their various choices of EOS that have different predictions for the relative size of the crust.

\subsection{Force Analysis}

The time evolution of the radial forces acting on the fluxoids is shown in Fig. 3, for the A1 model (corresponding to the results in Fig. 1 and Fig. 2a). One should note that a fluxoid, being a vortex, responds to a force by moving in a direction perpendicular to the force; a radial force does not affect its radial motion, directly. However, because of the dependence on $v_{\mathrm{p}}$ in Eq. 12 (which originally describes the azimuthal motion of the fluxoids) a "driving", or a "braking" role in flux expulsion might be assigned to an outward directed (positive), or an inward directed (negative) force, respectively.

As is seen in Fig. 3, the pinning force $F_{\mathrm{n}}$ is negative (directed inward) during the reverse creep phase and also the later part of the co-moving phase. Nevertheless, the major predicted flux expulsion does occur (see Fig. 1 and Fig. 2a) during the co-moving and, particularly, the reverse creep phases. This means that the dominant "driving" force for the flux expulsion is the buoyancy force which is positive throughout the evolution. And, that the overall role of the pinning force in the field decay of solitary pulsars is more like a "brake" preventing the flux to be expelled too rapidly. We have further verified this conclusion, which seems to be obvious from the results in Figs 1-3, by other tests of the model calculations where we have tentatively set either of the two forces equal to zero. Our conclusion about the braking role of the pinning force is, however, in contradiction with that of DC93 who attribute a driving role to this force throughout their paper, and make further statements which are not hence justified. The braking role of the pinning force is also in sharp contrast with the view adopted by Srinivasan et al. (1990) about the flux expulsion mechanism, eventhough comparison with their (SIF) model, in this regards, might be misleading and unjustified since their treatment does not address the dynamics to begin with.

The opposing role of the pinning force against the fluxoids outward migration, at late times $\left(\gtrsim 10^{7} \mathrm{yr}\right)$, is indeed true for all the FBE models adopted here. Except for including the pinning force these models are, otherwise, similar to the earlier dynamical studies of flux expulsion (Muslimove \& Tsygan 1985; Harvey et al. 1986) in having the buoyancy force as the driving cause of the flux expulsion, as verified above. It is for the role of the pinning force that the predicted field of a neutron star, for all of the FBE models, may never decay to very small values $\left(<10^{8} \mathrm{G}\right)$ even after very long times $\left(\gtrsim 10^{10} \mathrm{yr}\right)$. This is a fundamental difference which is much needed to account for the low field pulsars (to be discussed in the next section) that could not be possibly explained by the earlier flux expulsion scenarios which neglected the pinning.

We hoped to be able to comment about the relevance of the alternative physical assumptions discussed for the FBE models by comparing their predictions. However the predicted field evolution behaves very similarly in the different models, hence no further conclusions may be drawn. The reason for this rather unfortunate finding may be retraced to the braking role of the pinning force, $F_{\mathrm{n}}$, as well as the effective period of the core field expulsion; both discussed earlier. Models A1, B1, and DCC differ from the other two (see §3) in their prescription for calculating the magnitude of $F_{\mathrm{n}}$. Due to the dependence of $F_{\mathrm{n}}$ on $B_{\mathrm{c}}$ and $P_{\mathrm{s}}$ its predicted value for the two classes of models is markedly different only during the early short phase 
of forward creeping (see, also, Fig. 3.4 in Jahan-Miri 1996b). However, the major field expulsion occurs during the later prolonged phases, ie. when $F_{\mathrm{n}}$ acts as a brake and its predicted value is not much different for the different models. Hence, the distinction among the models with respect to $F_{\mathrm{n}}$ is, in effecte, washed out. A second distinction among the models (models A versus B) relates to their different prescriptions for calculating the curvature force, $F_{\mathrm{c}}$, which differ only when the fluxoid velocity is large $\left(\gtrsim v_{\max }\right)$. Large fluxoid velocities, which occurs only when neutron vortices too move fast, hence large differences between the value of $F_{\mathrm{c}}$ among the models takes place again only during the early phase of rapid spinning down of a single pulsar. Thus, in short, for the spin-down history of single pulsars the calculated forces on fluxoids at times $>1 \mathrm{Myr}$ are not much different for the different models treated here, hence resulting in similar field evolutions. The distinction among the predicted field evolutions due to the different FBE models could however be, in principle, quite significant as is encountered in the case of spin histories of neutron stars in close binaries which is discussed below.

\subsection{Observational Implications}

\section{Pulsar distribution:}

The evolutionary tracks for single pulsars on the spin-magnetic field diagram as predicted by SIF and DCC (the latter being typical for all the FBE models) are plotted in Fig. 4, for the different assumed initial field strengths. Points corresponding to the given ages of the neutron star are also marked along each track. As is seen in Fig. 4, the predicted final strength of $B_{\mathrm{s}}$, for FBE models, is found to depend sensitively, and inversely, on its initial value. This is a consequence of the direct correlation between the total expelled flux and the initial value of $B_{\mathrm{s}}$, which is expected because larger values of $P_{\mathrm{s}}$ are achieved for larger initial $B_{\mathrm{s}}$ values, as indicated earlier. However the final value of $B_{\mathrm{s}}$ is insensitive (not shown in Fig. 4) to the assumed initial values of $P_{\mathrm{s}}$ and $B_{\mathrm{c}}$, for changes in these quantities by almost two orders of magnitudes. In contrast, for the SIF model the final value of $B_{\mathrm{s}}$ is found to depend on the initial values of $P_{\mathrm{s}}$ and $B_{\mathrm{c}}$, as well as on the initial $B_{\mathrm{s}}$ (Fig. 4) eventhough having a direct correlation in this case. These correlations, for the SIF model, are in accord with the assumed relation $\frac{\dot{B}_{\mathrm{c}}}{B_{\mathrm{c}}}=-\frac{\dot{P}_{\mathrm{s}}}{P_{\mathrm{s}}}$, corresponding to $v_{\mathrm{p}}=v_{\mathrm{n}}$ at all times. A further point to note in Fig. 4, is the power-law time behavior of the field evolution of single pulsars at late times, which is realised for the lower initial fields. This feature which is common among the SIF and the FBE models, and was also pointed out by Srinivasan et al. (1990), has new observational implications which have been previously highlighted (Jahan-Miri 1996a).

\section{Very old neutron stars :}

In spite of the recent discovery of large redshifts for some $\gamma$-ray burst sources the association of a sub-class of them with a galactic population of highly magnetic old neutron stars (see, eg., Blandford 1992) may still be viable. While such an identification of the $\gamma$-ray bursters does not seem to be consistent with the predictions of FBEs for the field strengths of very old single neutron stars, it could be however accommodated by the SIF model. According to SIF very old neutron stars (with ages $\sim 10^{10}$ ) are expected to have rather large magnetic fields in the range $2 \times 10^{10} \lesssim B_{\mathrm{s}}<2 \times 10^{11} \mathrm{G}$, while the FBE models predict values of $B_{\mathrm{s}} \lesssim 2 \times 10^{10} \mathrm{G}$ for such stars (see Fig. 4). The FBE-predicted upper limit for the final fields is even smaller than above, $B_{\mathrm{s}}<3 \times 10^{9} \mathrm{G}$ at an age $\gtrsim 10^{8} \mathrm{yr}$, for the large initial values of $B_{\mathrm{s}} \gtrsim 10^{12.5} \mathrm{G}$. Furthermore, while the above results from Fig. 4 are for a value of $\tau=10^{7} \mathrm{yr}$, still smaller final fields would be the case for larger values of $\tau$ (compare Fig. 2a with Fig. 2b).

In contrast, DC93 suggested that for a neutron star having a magnetic axis aligned with its rotation axis the core field, according to the DCC model, would not be expelled even on large time scales $\left(>>10^{7} \mathrm{yr}\right)$, 
unlike the general case presented in Fig. 4. Thus, they concluded that the earlier proposed model of Ruderman \& Cheng (1988) for the burst sources being aligned neutron stars is consistent with a field evolution according to their (DCC) model. However, their conclusion has to be dismissed since it is based on the unusual assumption that the spin-down torque of a pulsar is only due to its magnetic dipole radiation. The usual and long lived consensus which is commonly adopted also for an observational determination of the strengths of the surface fields of pulsars is that the spin-down torque, due to combined effects of dipole radiation and outflow of relativistic particles, is independent of the inclination angle (see, eg., Manchester \& Taylor 1977, pp. 176-180; Srinivasan 1989).

Nevertheless, since a discussion of the predicted field decay by the FBE models for a tentatively assumed case of little spin-down torque acting on a neutron star serves to further elucidate the nature of the models we will pursue the discussion. Indeed, if the spin-down rate of a neutron star is assumed to be small the core field according to the FBE models would not be expelled much, as suggested by DC93. This happens mainly because a reverse creep phase does not occur in this case since the associated value of $\left|F_{\mathrm{n}}\left(\omega=-\omega_{\mathrm{cr}}\right)\right|$ would be too large. The large value of $F_{\mathrm{n}}$ follows from its inverse proportionality on $P_{\mathrm{s}}$ (see Eq. 2), and the fact that the assumed small spin-down torque result in small final values of $P_{\mathrm{s}}$. However, Ding et al. argued (see the last equation in DC93) that the large value of $F_{\mathrm{n}}$ in this case is a consequence of the direct proportionality of $\omega_{\mathrm{cr}}$ on $B_{\mathrm{c}}$, and the large value of $B_{\mathrm{c}}$. We argue that the dependence of $\omega_{\text {cr }}$ on $B_{\mathrm{c}}$ is irrelevant since $\left|F_{\mathrm{n}}\left(\omega=-\omega_{\mathrm{cr}}\right)\right| \propto 1 / B_{\mathrm{c}}^{\frac{1}{2}}$, which implies a smaller $\left|F_{\mathrm{n}}\right|$ for the assumed larger $B_{\mathrm{c}}$. In fact the reverse creep phase starts always at a large value of $B_{\mathrm{c}}$ even in (the non-aligned) cases where a substantial field decay does occur, as might be expected from the above dependence of $F_{\mathrm{n}}$ on $B_{\mathrm{c}}$. We conclude that the FBE-predicted little flux expulsion for the "aligned" case is only because $P_{\mathrm{s}}$ could retain its assumed small initial value, as the following test verifies. The two cases of field evolution presented in Fig. 5 are both for an assumed very small spin-down torque (ie. that expected due only to the dipole radiation for an inclination angle of $1 \mathrm{deg}$ ) but for two different initial values of $P_{\mathrm{s}}$. Eventhough in both cases $B_{\mathrm{c}}$ is large before the transition to the reverse creep phase, however substantial flux expulsion does takes place in the case where $P_{\mathrm{s}}$ is large. Note that in this latter case the reverse creep phase starts from the very beginning and persists throughout the evolution of the star. This clearly verifies the above mentioned role of $P_{\mathrm{s}}$ in determining the conditions for occurrence of a reverse creep phase. In addition, the above arguments indicate a potential possibility for having very old neutron stars with strong magnetic fields, in the context of the FBE models too. This may be achieved provided the star is not spun down to large periods $\gtrsim 1 \mathrm{~s}$, independent of whether or not the surface field is aligned with the rotation axis of the star, as it also happens to some extent for the low field pulsars shown in Fig. 4.

\section{Active lifetimes of pulsars :}

The radio-active lifetimes (defined by the condition $\frac{B_{\mathrm{s}}}{P_{\mathrm{s}}^{2}}=0.2 \times 10^{12}$ ) of pulsars, calculated for the predicted spin-field evolution in the different FBE models, are shown in Fig. 6 against the initial field strengths, for the two values of $\tau=10^{7}$ and $10^{8} \mathrm{yr}$, separately. Corresponding curves for the case of exponential field decay model, labeled as "Exp.", (which assumes the total surface field decays exponentially, with no constraint, on the given time scale $\tau$ ) are also included, for comparison. The predicted lifetimes by the different FBE models are very similar as can be seen in Fig. 6 (this is true also for the A2 and B2 models which have been omitted for the clarity), again making any attempt for a selection among the five FBE models fruitless. Nevertheless, there exist a marked difference (in Fig. 6) between the results of FBEs, as well as SIF, in contrast to those of "Exp.", in particular for values of $\tau \lesssim 10^{7}$ yr. This difference should, in principle, have testable consequences for studies of pulsar statistics to decide between the flux expulsion scenario, in general, and the exponential model, for the single pulsars (see Jahan-Miri, 1996a, for further discussion). 


\section{Binary Evolution Models}

The spin evolution of a neutron star in a binary system with a mainsequence star is expected to be different from that of a single pulsar. The interaction of the neutron star magnetosphere with the stellar wind of the companion star could result in final large values of $P_{\mathrm{s}} \sim 10^{4}-10^{5} \mathrm{~s}$, in contrast to the much smaller values achieved in the case of single pulsars. Magnetic evolution of binary neutron stars as predicted by the flux expulsion models (FBEs and SIF) is therefore expected to be, in principle, quite different than that of the single pulsars. In this section we employ the FBE models, for the first time, in a study of the field evolution of neutron stars in binaries. We consider models for the orbital and spin evolution of a neutron star, of a mass $M_{\mathrm{n}}$, born in a binary with an orbital period $P_{\text {orb }}$, corresponding to an orbital separation $a$. The binary companion of the neutron star is a mainsequence star of mass $M_{2}$ which loses mass in the form of a spherical uniform stellar wind at a rate $\dot{M}_{2}$. In the picture of flux expulsion models the evolutions of the spin period and the magnetic field of the neutron star in such a binary would be intimately coupled. While the spin-down process would tend to reduce the field strength, the reduced field strength (together with the increased spin period) will in turn affect the rate and the direction of the spin variations. We follow this coupled evolution of the surface magnetic field and the spin period of the neutron star for a time of the oreder of the expected mainsequence lifetime of the companion star. The orbital and spin evolutions for the expected Roche-lobe overflow phase is not however simulated. Nevertheless, it should be noted that the computed field evolution of the recycled pulsars is not affected by this omission of the Roche-lobe phase of the binary evolution. The latter spin-up phase of a recycled pulsar would have no direct effect on the flux expulsion, hence no consequences for the field evolution, except for the general decay of the crustal field which is accounted for in our computations. Thus our simulations are complete, within the limitations of the models, as far as the field evolution of recycled pulsars is concerned; a point which has been overlooked by some authors (Urpin et. al. 1998) in referring to the earlier cited binary evolution simulations based on the SIF model.

We assume that the first phase of the binary evolution, namely the active-pulsar phase with a dipole spin-down (during which the neutron star spins down due only to the dipolar radiation torque on it), lasts till the ram pressure of the stellar wind overcomes the pressure of the "pulsar wind" at the accretion radius (see, e.g., Illarionov \& Sunyaev 1975; Davies \& Pringle 1981). During this period the stellar wind will have no dynamical effect on the neutron star. The pulsar's core magnetic field will undergo an expulsion determined by the dipole spin-down rate, according to the FBE models. In the subsequent two phases where the accreted wind matter interacts directly with the magnetosphere we assume that a steady Keplerian disk is formed by the accretion flow outside the magnetosphere, with the same sense of rotation as that of the neutron star. This is the least efficient configuration for angular momentum extraction from the neutron star which we have considered, in addition to the more efficient geometries such as a spherically symmetric radial infall.

The accretion flow interacts with the magnetosphere at its characteristic boundary radius $R_{\text {mag }}$, which is defined by the condition of balance between the magnetic pressure and the ram pressure of infalling flow (Davidson \& Ostriker 1973):

$$
R_{\text {mag }}=1.88 \times 10^{-12}\left(\frac{B_{\mathrm{s}}^{2}}{\dot{M}_{\text {acc }}}\right)^{2 / 7} R_{\odot}
$$

where $\dot{M}_{\text {acc }}$ is the rate of capture of wind matter, in units of solar masses per year, as defined in Jahan-Miri \& Bhattacharya (1994; Eq. 4 therein). This interaction spins the neutron star up or down depending on the sign of the quantity $V_{\text {dif }}\left(=V_{\text {corot }}-V_{\text {Kep }}\right)$ evaluated at the boundary of the magnetosphere. Here, $V_{\text {corot }}$ is the speed of co-rotation with the neutron star at a given distance from it, and $V_{\text {Kep }}$ is the Keplerian speed at the same distance. In the limiting case when the co-rotation velocity $V_{\text {corot }}$ becomes equal to the Keplerian velocity $V_{\text {Kep }}$ the neutron star will conserve its spin period while accretion onto the star will continue. The rate $\dot{L}_{\mathrm{s}}$ of transfer of angular momentum between the stellar wind and the neutron star is 
assumed to be equal to $\dot{M}_{\text {acc }}$ times a specific angular momentum corresponding to the difference between the co-rotation velocity with the neutron star and the Keplerian velocity evaluated at a distance $R_{\text {mag }}$ from the neutron star. In general, then,

$$
\dot{L}_{\mathrm{s}}=\eta \times V_{\mathrm{dif}} \times R_{\mathrm{mag}} \times \dot{M}_{\mathrm{acc}}
$$

will be used, where $\eta$ is the efficiency factor included to take into account the uncertainties due to the detailed geometry of the interaction and the actual value of the specific angular momentum carried by the accreted wind just before and after the interaction. Different rates of angular momentum transfer are thus tested by assuming different values for $\eta$ while $\eta=1$ corresponds to the case of disk-accretion with a

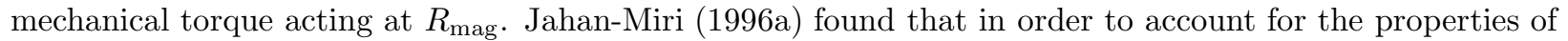
binary pulsars evolved in both massive as well as low-mass systems, simultaneously and self-consistently, larger values of $\eta>1$ were preferred. Hence, and in contrast to the adopted range of values between $0.2-1.0$ for the efficiency factor in Jahan-Miri \& Bhattacharya (1994), here we use the larger values of $\eta$; see below.

The following coupled differential equations for the time evolution of $M_{\mathrm{n}}, a, P_{\mathrm{s}}$, along with those for $B_{\mathrm{c}}$ and $B_{\mathrm{s}}$ discussed before ( $(3)$, are solved numerically, for the various combinations of parameter values indicated below.

$$
\begin{aligned}
\frac{\mathrm{d} a}{\mathrm{~d} t} & =2 a\left\{\left.\frac{\dot{L}_{\text {losses }}}{L_{\mathrm{orb}}}\right|_{\text {losses }}-\frac{\dot{M}_{2}}{M_{2}}\left[1+(\alpha-1) \frac{M_{2}}{M_{\mathrm{n}}}-\frac{1}{2} \alpha \frac{M_{2}}{M}-\alpha \beta \frac{M_{\mathrm{n}}}{M}\right]\right\} \\
\frac{\mathrm{d} M_{\mathrm{n}}}{\mathrm{d} t} & =\left\{\begin{array}{lll}
\dot{M}_{\mathrm{acc}} & \ldots \ldots \ldots \ldots & \text { accretion phase } \\
0.0 & \ldots \ldots \ldots \ldots & \text { propeller phase }
\end{array}\right. \\
\frac{\mathrm{d} P_{\mathrm{s}}}{\mathrm{d} t} & =3.18 \times 10^{-3} \eta\left(\frac{\dot{M}_{\mathrm{acc}}}{M_{\odot} \mathrm{yr}^{-1}}\right)\left(\frac{R_{\mathrm{mag}}}{\mathrm{km}}\right)\left(\frac{P_{\mathrm{s}}}{\mathrm{s}}\right)^{2}\left(\frac{V_{\mathrm{dif}}}{\mathrm{km} \mathrm{s}^{-1}}\right) \mathrm{s} \mathrm{yr}^{-1}
\end{aligned}
$$

where $L_{\text {orb }}$ is the orbital angular momentum, $\dot{L}_{\text {losses }}$ is the rate of change in $L_{\text {orb }}$ except for the contribution due to the escaping matter from the system which is already taken into account, $M=M_{\mathrm{n}}+M_{2}$ is the total mass of the binary, $\alpha$ is the ratio of the mass loss rate from the system to that from the secondary, and $\beta$ is the ratio of the effective specific angular momentum of the escaping matter to that in the companion star. As indicated earlier, a spin-up phase of the neutron star would have no effect on the flux expulsion out of its core, and the core field remains constant during such a period of time. Also, note that during the active pulsar phase $\dot{P}_{\mathrm{s}}$ will be given by the relation indiacted earlier ( $\left.\S 4\right)$ for the single pulsars, instead of Eq. 17 which is applicable for the other two phases of magnetospheric interaction with the accreted matter; see Jahan-Miri \& Bhattacharya (1994), and Jahan-Miri (1996a), for more details of the binary evolution models. The computations were repeated using different combinations of the following values of the parameters and the initial conditions, for each of the FBE models, separately:

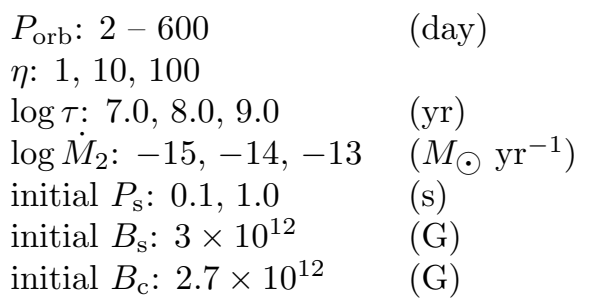

The evolution of a neutron star in a binary with a low-mass companion $\left(M_{2}=1.0 M_{\odot}\right)$ is followed for a period of $10^{10} \mathrm{yr}$ and its final surface field is determined, in order to be compared with the observed fields of low mass binary, and millisecond, pulsars. 


\section{Predicted Fields of Recycled Pulsars}

The general features of the computed evolution for the outward velocities of the fluxoids and the vortices, as well as the rotational lag, are similar to that descibed earlier in the case of single pulsars. A new feature is the rapid increase in the fluxoids velocity during the reverse creep phase, which is expected because of the enhanced spinning down of the star in a close binary. The distribution of the final surface field strengths versus the initial orbital periods are plotted in Fig. 7, as predicted by two of the FBE models, for the given parameter values. Fig. 7 shows that the observed magnetic field strengths of the low-mass recycled pulsars, and millisecond, pulsars may be successfully reproduced by the FBE models. In addition, the observational data on eight low-mass binary pulsars, which are expected to have been recycled in low-mass binary systems, are also presented in Fig. 7. The measured orbital periods for these systems have been corrected for the expected change in the period during a final Roche-lobe overflow mass transfer phase in the binary to infer the corresponding intial values of $P_{\text {orb }}$, listed in Table 1, for use in Fig. 7 .

Table 1- The observed low-mass binary pulsars

\begin{tabular}{cccccc}
\hline PSR & $\begin{array}{c}P_{\mathrm{s}} \\
(\mathrm{msec})\end{array}$ & $\begin{array}{c}P_{\text {orb }} \\
(\text { day })\end{array}$ & $\begin{array}{c}M_{2} \\
\left(M_{\odot}\right)\end{array}$ & $\begin{array}{c}\log B_{\mathrm{s}} \\
(\mathrm{G})\end{array}$ & $\begin{array}{c}\text { initial- } P_{\text {orb }} \\
(\text { day })\end{array}$ \\
\hline $0820+02$ & 864 & 1232 & $0.2-0.4$ & 11.48 & 300 \\
$1953+29$ & 6.13 & 117 & $0.2-0.4$ & 8.63 & 12 \\
$1855+09$ & 5.36 & 12.3 & $0.2-0.4$ & 8.48 & 1 \\
$\mathrm{~J} 1713+0747$ & 4.57 & 67.83 & $0.3-0.5$ & 8.28 & 6 \\
$\mathrm{~J} 2019+2425$ & 3.93 & 76.51 & $0.3-0.5$ & 8.26 & 7 \\
$\mathrm{~J} 1643-1224$ & 4.6 & 147 & 0.14 & 8.6 & 24 \\
$\mathrm{~J} 1455-3330$ & 8.0 & 76 & 0.3 & 8.3 & 10 \\
$\mathrm{~B} 1800-27$ & 334.4 & 406.8 & 0.15 & 10.9 & 60 \\
\hline
\end{tabular}

References: Bhattacharya and van den Heuvel 1991; Foster, Wolszczan and Camilo 1993;

Nice, Taylor and Fruchter 1993; Johnston et al. 1995; Lorimer et al. 1995.

Within the uncertainties associated with the value of $\dot{M}_{2}$, which could be also varying with time, and the other unknown parameters of the binary pulsars, the computed curves in Fig. 7 seem to agree with the data points. It is also understood that while the curves in Fig. 7 represent a particular choice of the values for the pulsar-binary parameters however the true value of each of them might have been quite different among the corresponding eight systems. Qualitatively similar agreement with the data, as in Fig. 7, is obtained also for many other choices of the parameter values, as well as for the other FBE models, namely A2, B1, and B2. Nevertheless, and in contrast to the case of single pulsars, the predicted field evolution of a given binary pulsar is found to be quite different according to the different FBE models. This is promissing, in the sense that it offers a potential possibility not accessible from the application of the models to single pulsars. One might hope to distinguish among the models and gain insight into the interior physics of neutron stars, based on a comparison of the predicted spin-field evolution with the observational data on the recycled systems. However, we have not been able to pinpoint any preferences among the various flux expulsion models, at this stage, because of the uncertainities due to the free and unknown parameters (ie. 
$\eta, \dot{M}_{2}, \tau$, etc.) which are encountered even in a simplified treatment of the binary evolution that we have used. That is, within the indicated ranges for the parameter values, all FBE models merit the same success in comparison to the existing observational data on the recycled pulsars.

Nevertheless, the above general success of FBE, and SIF, models which has not been so far reported for any other field decay model of neutron stars, provides a strong support for the flux expulsion scenario. It is further noted that the SIF model was shown previously to be consistent with the data also in the case of pulsars recycled in binaries with massive companion stars (Jahan-Miri 1996a). Thus, judging on the overall agreement seen here between the predictions of SIF with that of FBE models, in the case of single and low-mass binary pulsars, it might be justified to generalize the success of SIF for the case of massive binaries to FBE models as well. On the other hand, the other existing field evolution model of neutron stars, which assumes the total magnetic flux to be confined to the crust, has been also shown to result in the case of binary pulsars to a decay of the field down to values similar to that of the recycled pulsar (Geppert et. al. 1996; Urpin et. al. 1998). However, the spin-orbital binary evolution have not been really followed in these studies in all its details, and instead general estimated values for the rate and duration of accretion of matter onto the neutron star have been used. More importantly, the dependence of the final fields on the orbital periods which is shown in Fig. 7 for the FBE models and is also compared with the observational data remains to be demonstrated for the model of crustal field decay due to accretion.

As in the case of single pulsars, for neutron stars evolved in many of the binary systems which we have simulated, again the pinning force on fluxoids acts as an obstacle against an otherwise more rapid and enhanced flux expulsion. Indeed, we have verified that setting $F_{\mathrm{b}}+F_{\mathrm{c}}=0$ in the models, namely having $F_{\mathrm{n}}$ as the only existing driving force, results in a much smaller flux expulsion than otherwise. In contrast, if $F_{\mathrm{n}}=0$ is adopted practically zero final field values are obtained, which further demonstrate the braking role of $F_{\mathrm{n}}$. Nevertheless, the essential role played by the pinning force in the field evolution of recycled old pulsars may not be overlooked. A model which discards the pinning force and relies only on the buoyancy is obviously unable to account for any flux to be present in the cores of such old pulsars. In contrast, the FBE (and SIF) models not only account for the observed fields of the old binary and millisecond pulsars, they also predict a correlation between the final field strength of a recycled pulsar and its spin period history, by virtue of the role of the pinning force.

\section{Summary and Conclusions}

We have studied a scenario for the evolution of the magnetic fields of neutron stars, assuming that the magnetic flux resides in the proton superconductor core of the star and is carried by the fluxoids. We have employed a dynamical treatment for the flux expulsion trying to improve our earlier studies based on the original model which adopted an expulsion rate equal to the spin-down rate of the star. In the present work, the rate of expulsion of the flux out of the core is determined by explicitly calculating the radial velocity of the fluxoids which are subject to various forces. We have included forces due to pinning between the fluxoids and the neutron superfluid vortices, buoyancy, scattering of electrons, and tension of the flux

lines. Alternative possibilities for evaluation of these forces were considered. The predictions of the various corresponding field decay models for the evolution of single and binary pulsars were discussed, and our conclusions are further summarized below.

- The explored flux expulsion models predict a restricted decay of the magnetic field of pulsars that also accounts for the residual fields of the very old neutron stars. The pinning force plays two opposite and essential roles in the magnetic evolution of the star. The success of the models in predicting the residual fields of the recycled binary and millisecond pulsars is because of the braking role of this force against the fluxoids outward motion. This effect also result in a long-lived slowly evolving 
phase, hence an increased radio-active lifetime, for some of the single pulsars. However, the pinning force also has a positive role in driving the fluxoids out of the core which is revealed in the predicted dependence of the final field strengths of the recycled pulsars on the binary parameters that determine the spin history of the star.

- Flux expulsion under the influence of the buoyancy force alone, in the absence of the pinning force, leads to vanishing field strengths in old binary and millisecond pulsars, hence it is definitely ruled out. In contrast, a (tentative) model which invokes the pinning force and neglects the buoyancy would result, in general, in larger final fields than in the presence of the both forces; however, for some choices of the parameters the final fields might be small as well.

- The alternative dynamical models, which we have considered for the motion of fluxoids and vortices in the core of neutron stars, result in similar predicted spin-magnetic evolutions for the single pulsars. For the binary pulsars, eventhough the predictions of the models are quite different in many of the cases, however similar acceptable results are still obtained for each of them, albeit for the different plausible values of the parameters. Hence, the underlying assumptions in these models remain observationally equivalent. The ideas which we entertained in these models are a) a neutron vortex remains straight while moving, versus, its pinned segments might creep independently, $b$ ) a fluxoid may bend and be reacted by its tension, versus, the collective rigidity of the lattice prevents any bending, c) fluxoids are bent outward (inward) at all times smaller (larger) than the field decay time scale in the crust, versus, fluxoids are bent outward when moving faster than the permitted motion of their end points due to diffusion of the flux in the crust and remain straight otherwise.

- The role of the coherent scattering of the electrons by the fluxoid lattice does not seem to be fully understood. Flux expulsion in the presence of the coherent scattering might depend on the electron currents across the core-crust boundary. Assuming that the associated maximum radial velocity of the electrons is similar to the limiting velocity of the fluxoids in the case of collective rigidity of their lattice, we speculated that our models for the case of collective rigidity would represent the case of coherent scattering too.

- In contrast to the dynamical models which we used to determine the time evolution of the fluxoids velocity, the original spin-down induced flux expulsion model assumes equal velocities for the fluxoids and the vortices, at all times. The close agreement between its predictions and those of the dynamical models is largely because a substantial flux expulsion occurs during a co-moving phase of the two families of the vortices, even according to the latter models. This model, which offers a much simpler approach for a calculation of the field evolution compared to that employed here, might be further justified on the dynamical grounds. The co-moving phase corresponds to a less energy dissipation for the coupled system of the two families of vortices, than their crossing through each other which costs energy. It is feasible, though need to be demonstrated, that the more economical co-moving phase is preferred, and maintained at all times, by the magnetohydrodynamics of the interior quantum liquid; noticing also that the fluxoids as well as the vortices are both magnetized and are rooted in the same highly conductive medium at the bottom of the crust.

- Finally, an effective time scale in the range $10^{7}-10^{8}$ yr for the decay of the magnetic field in the crust of a neutron star is suggested, based on the preferred results of the model calculations presented here.

The author is grateful to the referee for the very useful comments and suggestions which helped to improve the text largely. I wish to thank G. Srinivasan for reading a separate earlier report of this work and making useful suggestions. I am in debt to D. Bhattacharya for use of the binary evolution code which was developed for our earlier work. I am grateful to Raman Research Institute for their kind hospitality, and for using their Computing facilities in carrying out this study. 


\section{REFERENCES}

Alpar M. A., Sauls J. A., 1988, ApJ, 327, 723

Bhattacharya D., Srinivasan G., 1991, in Ventura J., Pines D., eds, Proc. NATO ASI 344, Neutron Stars: Theory and Observation. Kluwer, Dordrecht, p. 219

Bhattacharya D., van den Heuvel E. P. J., 1991, Phys. Rep., 203, 1

Bhattacharya D., Datta B., 1996, MNRAS, 282, 1059

Blandford R., 1992, in Pines D., Tamagaki R., Tsuruta S., eds, Proc. US-Japan SENS '90, The Structure and Evolution of Neutron Stars. Addison-Wesley Pub., p. 104

Davidson K., Ostriker J. P., 1973, ApJ, 179, 585

Davies R. E., Pringle J. E., 1981, MNRAS, 196, 209

Ding K. Y., Cheng K. S., Chau H. F., 1993, ApJ, 408, 167

Flowers E., Ruderman M. A., 1977, ApJ, 215, 302

Foster R. S., Wolszczan A., Camilo F., 1993, ApJ, 410, L91

Geppert U., Urpin V., Konenkov D., 1996, A\&A, 307, 807

Goldreich P., Reisenegger A., 1992, ApJ, 395, 250

Harrison E., 1991, MNRAS, 248, 419

Harvey J. A., Ruderman M. A., Shaham J., 1986, Phys. Rev. D, 33, 2084

Jahan-Miri M., 1996a, MNRAS, 283, 1214

Jahan-Miri M., 1996b, PhD Thesis, Indian Institute of Science, Bangalore

Jahan-Miri M., 1998, ApJ, 501, L185

Jahan-Miri M., Bhattacharya D., 1994, MNRAS, 269, 455

Johnston S., Manchester R. N., Lyne A. G., Kaspi V. M., D́Amico N., 1995, A\&A, 293, 795

Jones P. B., 1987, MNRAS, 228, 513

Jones P. B., 1988, MNRAS, 233, 875

Jones P. B., 1991, MNRAS, 253, 279

Illarionov A. F., Sunyaev R. A., 1975, A\&A, 39, 185

Manchester R. N., Taylor J. H., 1977, Pulsars. W. H. Freeman \& Company, San Francisco

Lorimer D. R., Nicastro L., Lyne A. G., Bailes M., Manchester R. N., Johnston S., Bell J. F., D́Amico N., Harrison P. A., 1995, ApJ, 439, 933

Muslimov A. G., Tsygan A. I., 1985, Ap\&SS, 115, 43

Nice D. J., Taylor J. H., Fruchter A. S., 1993, ApJ, 402, L49

Phinney E. S., Kulkarni S. R., 1994, ARA\&A, 32, 591

Pines D., Alpar M. A., 1992, in Pines D., Tamagaki R., Tsuruta S., eds, Proc. US-Japan SENS '90, The Structure and Evolution of Neutron Stars. Addison-Wesley Pub., p. 7

Ruderman M., 1972, ARA\&A, 10, 427

Ruderman M., 1991, ApJ, 366, 261 
Ruderman M., 1995, in Fruchter A. S., Tavani M., Backer D. C., eds, Proc. Aspen Conference on Millisecond Pulsars: A Decade of Surprises. ASP Conference series vol. 72, p. 276

Ruderman M. A., Cheng K. S., 1988, ApJ, 335, 306

Sauls J. A., 1989, in Ögelman H., van den Heuvel E. P. J., eds, Proc. NATO ASI 262, Timing Neutron Stars. Kluwer, Dordrecht, p. 457

Sonin E. B., 1987, Rev. Mod. Phys., 59, 87

Srinivasan G., 1989, Astron. Ap. Rev., 1, 209

Srinivasan G., Bhattacharya D., Muslimov A. G., Tsygan A. I., 1990, Curr. Sci., 59, 31

Urpin V., Geppert U., Konenkov D., 1998, MNRAS, 295, 907

van den Heuvel E. P. J., 1994, in Nussbaumer H., Orr A., eds, Proc. Saas-Fee Advanced Course 22, Interacting Binaries. Springer-Verlag, Heidelberg, p. 263

This manuscript was prepared with the AAS LATEX macros v4.0. 
Fig. 1. - The top panel shows the predicted time evolution of the lag $\omega$ and its critical value $\omega_{\text {cr }}$ in a solitary neutron star according to the A1 model. The bottom panel shows the corresponding evolution of the velocities of the fluxoids $\mathrm{V}_{\mathrm{p}}$, and the vortices $\mathrm{V}_{\mathrm{n}}$. Initial values of $B_{\mathrm{s}}=10^{12.5} \mathrm{G}, B_{\mathrm{c}}=0.9 B_{\mathrm{s}}$, and a value of $\tau=10^{7}$ yr have been used.

Fig. 2.- The predicted time evolution of the strength of the magnetic field in the core $B_{\mathrm{c}}$ and at the surface $B_{\mathrm{s}}$, and the spin period $P_{\mathrm{s}}$ in a solitary neutron star according to the A1 model. The top panel (a) is for an assumed value of $\tau=10^{7} \mathrm{yr}$ and corresponds to the results in Fig. 1, while the bottom panel (b) is for $\tau=10^{8}$ yr.

Fig. 3.- The predicted time evolution of the various radial forces acting on fluxoids (per unit length) in a solitary neutron star, according to the A1 model and corresponding to the results in Fig. 1 and Fig. 2a. The three curves represent the pinning force $\mathrm{F}_{\mathrm{n}}$, the drag force $\mathrm{F}_{\mathrm{v}}$, and the sum of the buoyancy and curvature forces $\mathrm{F}_{\mathrm{b}}+\mathrm{F}_{\mathrm{c}}$.

Fig. 4.- Evolutionary tracks on the $B-P$ diagram for solitary pulsars born with the different assumed initial field strengths, as predicted in the different models discussed in the text. The results shown are for the SIF and the DCC models, while the latter is similar to those of the other FBE models. Positions of the neutron stars at various ages are marked on each track, and the spin-up line and the death line are also shown in each panel. Initial values of $B_{\mathrm{c}}=0.9 B_{\mathrm{s}}$, and a value of $\tau=10^{7}$ yr have been used.

Fig. 5.- Similar graphs as in Figs 1 and 2, but for the tentatively assumed case of a small spin-down torque acting on the neutron star (corresponding to the dipole radiation torque alone for a star with nearly parallel spin and magnetic axes). The two panels on the left are for an initial value of the spin period $P_{\mathrm{s}}=0.01 \mathrm{~s}$, while the right panels are for the case with initial $P_{\mathrm{s}}=2.0 \mathrm{~s}$. Notice that the velocity curves for $\mathrm{V}_{\mathrm{n}}$ and $\mathrm{V}_{\mathrm{p}}$ on the left panel coincide throughout.

Fig. 6.- The predicted radio-active lifetimes of single pulsars versus their initial surface fields, according to the different field decay models discussed in the text, while "Exp." represents the pure exponential decay model. Results for two different values of $\tau$ are shown in the two panels, as indicated.

Fig. 7.- Final values of the surface magnetic field strengths of neutron stars evolved in low-mass binaries versus initial orbital periods, as predicted in two of the FBE models. The other models (namely A2, B1, and B2) also produce similar results. The different curves, in each panel, correspond to the different assumed values of the companion mass-loss rate $\dot{M}_{2}$, as indicated by their logarithmic values, in units of $M_{\odot} \mathrm{yr}^{-1}$. 
Encircled dots represent observed binary radio pulsars that are descendants of wide low-mass binaries for which the initial orbital periods can be estimated. Initial values of $B_{\mathrm{s}}=3.16 \times 10^{12} \mathrm{G}, B_{\mathrm{c}}=0.9 B_{\mathrm{s}}, P_{\mathrm{s}}=0.1 \mathrm{~s}$, together with values of $\tau=10^{7} \mathrm{yr}$, and $\eta=10$ have been used. 

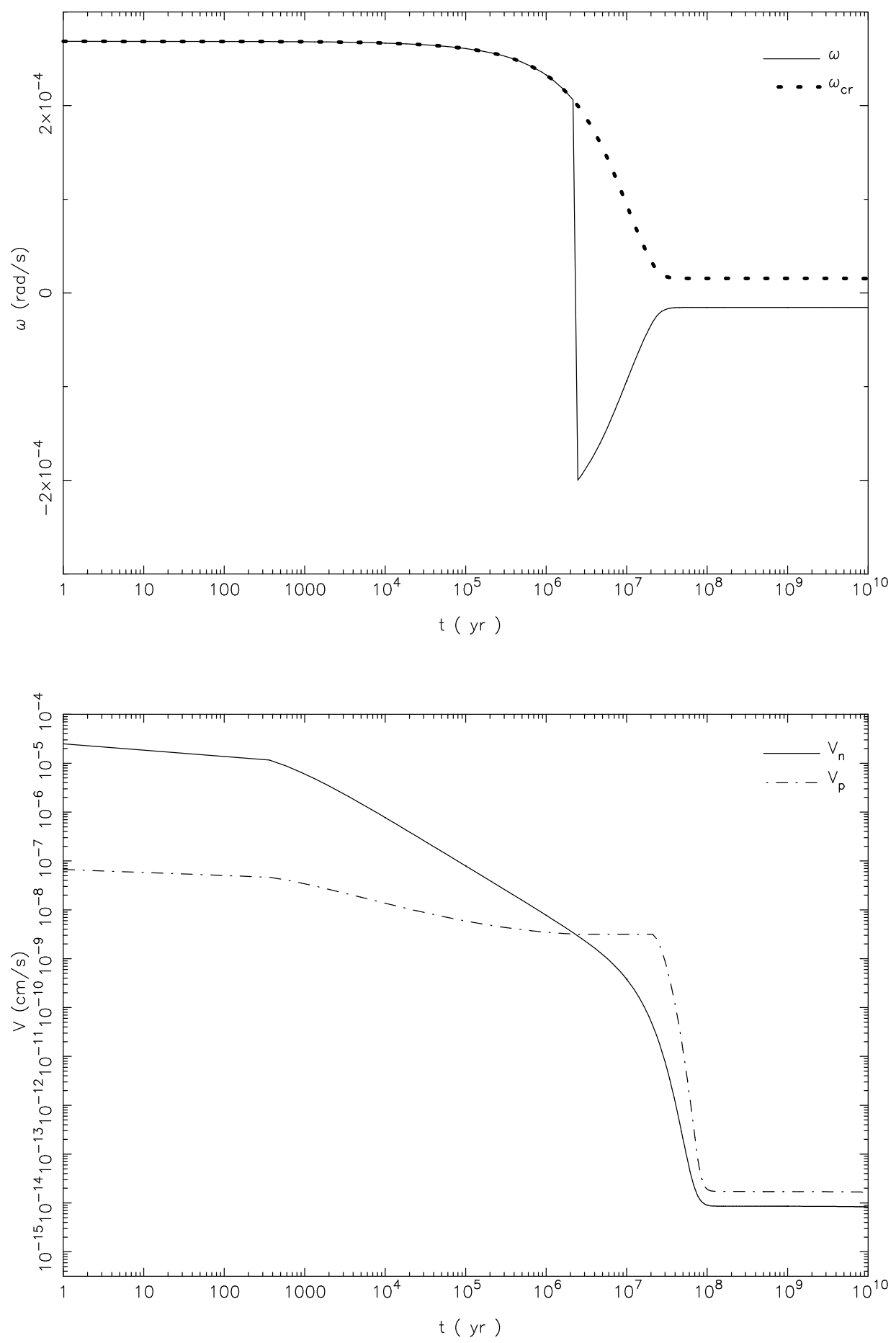

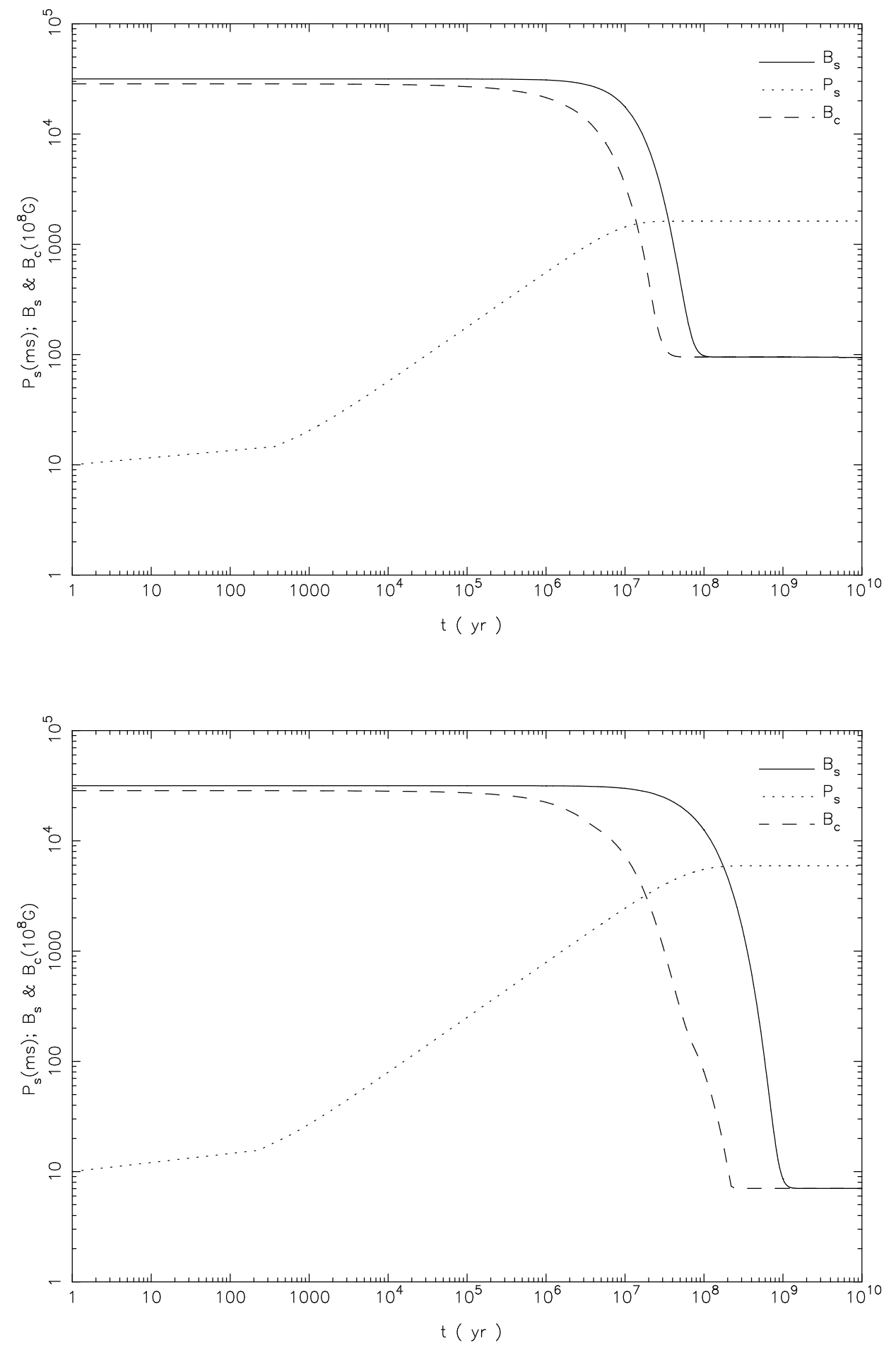


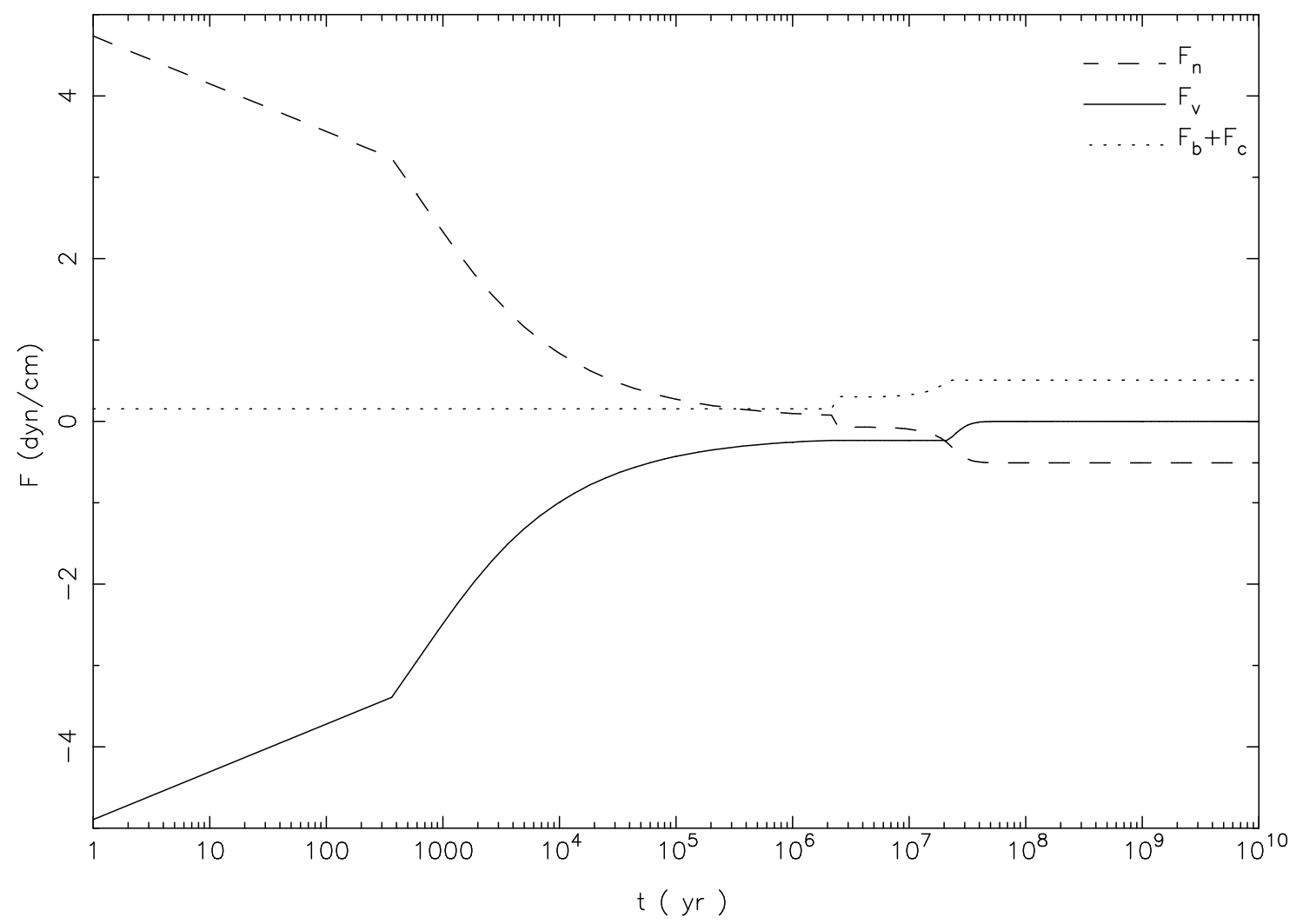




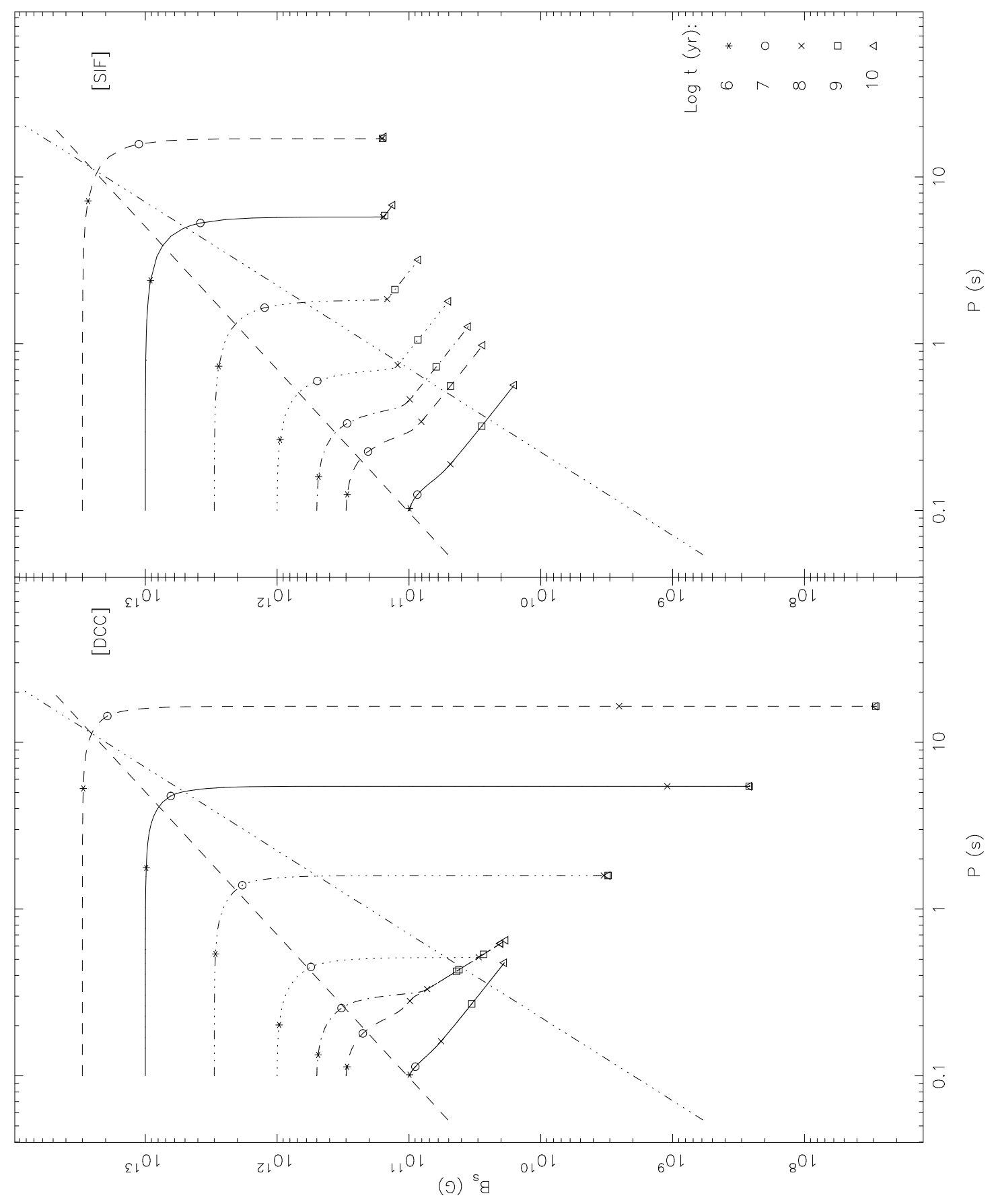



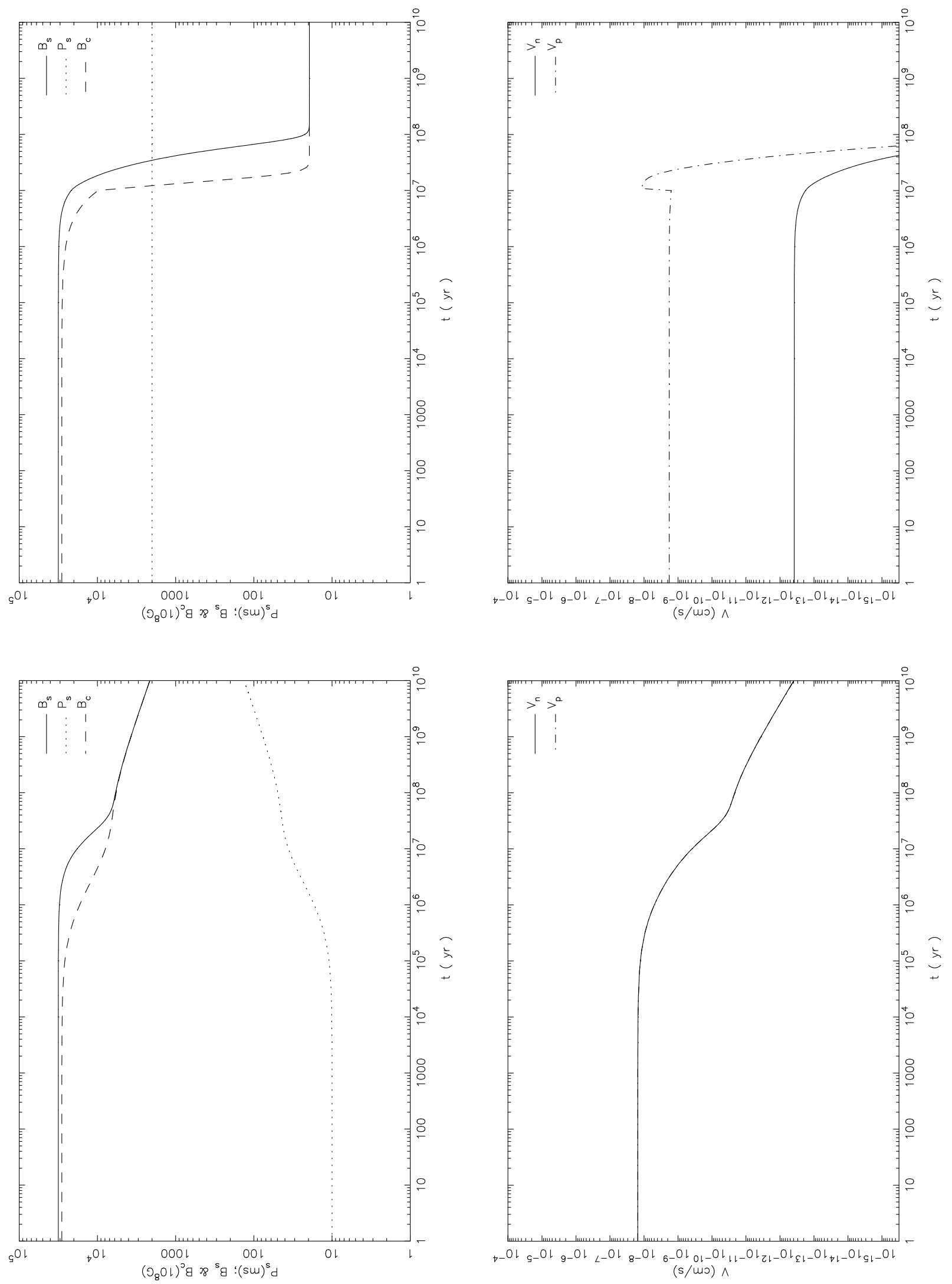


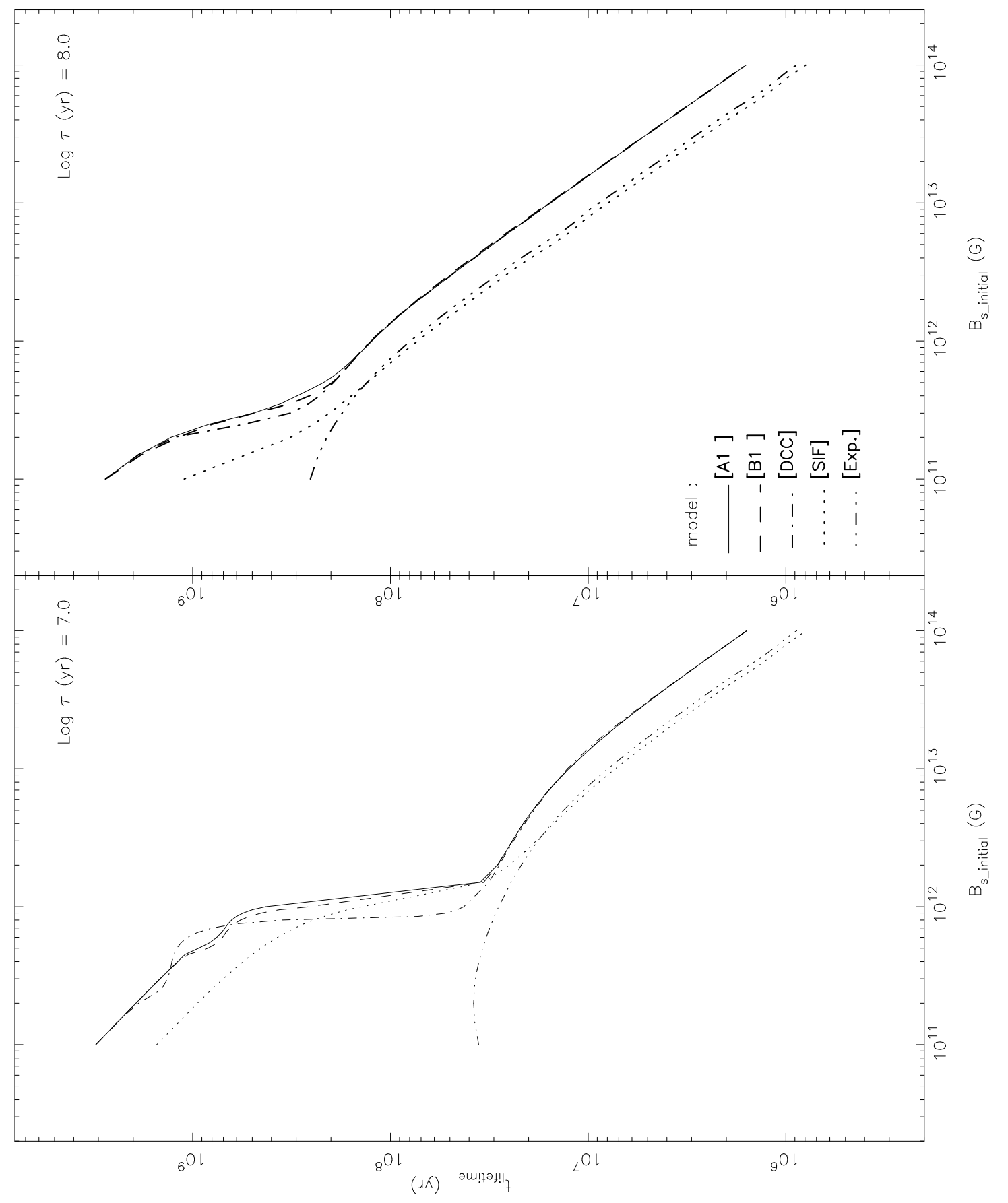



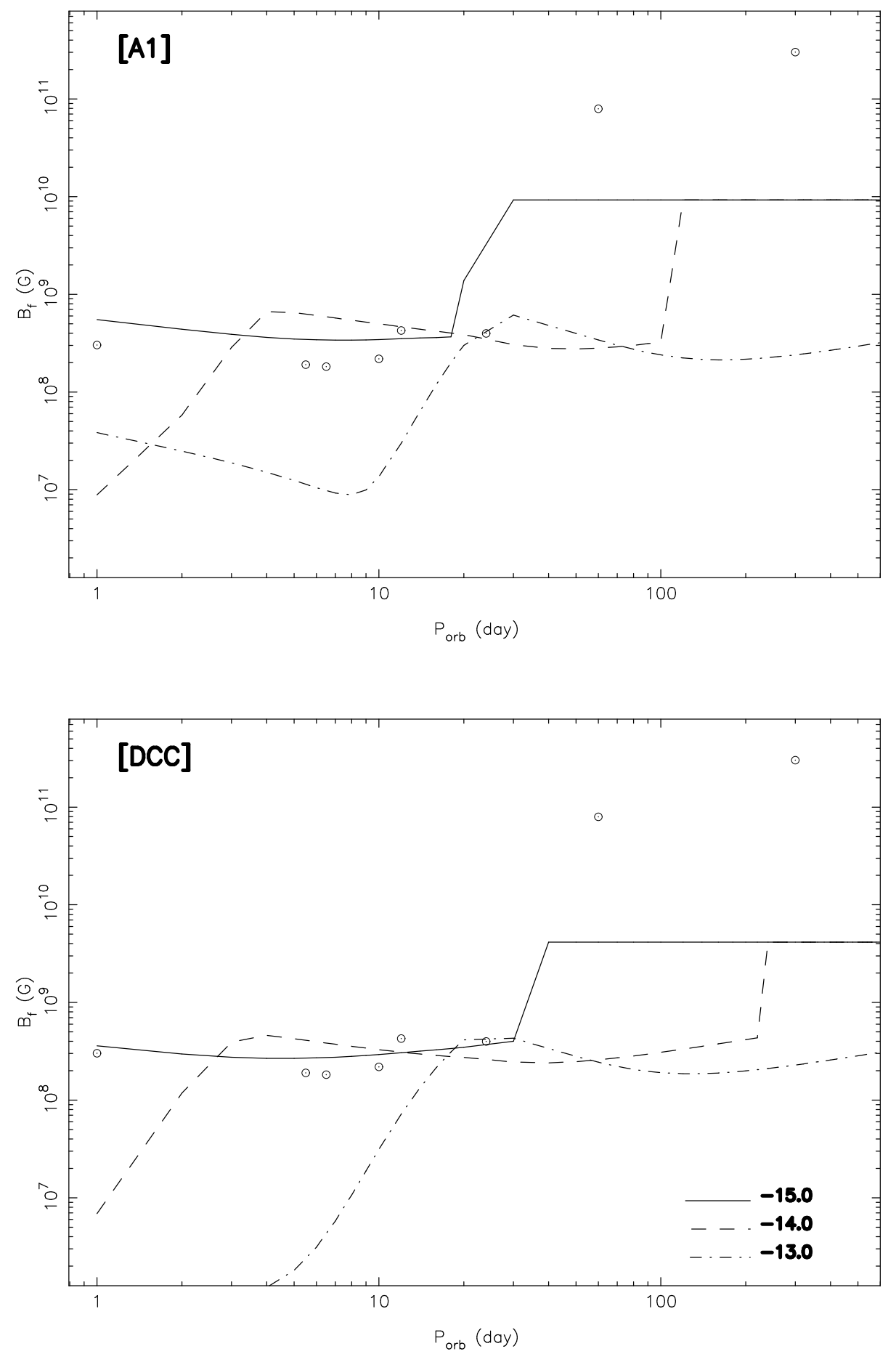